\title{
Hierarchical Unbiased Graph Shrinkage (HUGS): A Novel Groupwise Registration for Large Data Set
}

\author{
Shihui Ying ${ }^{\mathrm{a}, \mathrm{b}}$, Guorong Wu ${ }^{\mathrm{a}}$, Qian Wang ${ }^{\mathrm{a}, \mathrm{c}}$, and Dinggang Shen ${ }^{\mathrm{a},{ }^{*}}$ \\ aDepartment of Radiology and BRIC, University of North Carolina at Chapel Hill, NC 27599, USA \\ bepartment of Mathematics, School of Science, Shanghai University, Shanghai 200444, P. R. \\ China \\ 'Department of Computer Science, University of North Carolina at Chapel Hill, NC 27599, USA
}

\begin{abstract}
Normalizing all images in a large data set into a common space is a key step in many clinical and research studies, e.g., for brain development, maturation, and aging. Recently, groupwise registration has been developed for simultaneous alignment of all images without selecting a particular image as template, thus potentially avoiding bias in the registration. However, most conventional groupwise registration methods do not explore the data distribution during the image registration. Thus, their performance could be affected by large inter-subject variations in the data set under registration. To solve this potential issue, we propose to use a graph to model the distribution of all image data sitting on the image manifold, with each node representing an image and each edge representing the geodesic pathway between two nodes (or images). Then, the procedure of warping all images to their population center turns to the dynamic shrinking of the graph nodes along their graph edges until all graph nodes become close to each other. Thus, the topology of image distribution on the image manifold is always preserved during the groupwise registration. More importantly, by modeling the distribution of all images via a graph, we can potentially reduce registration error since every time each image is warped only according to its nearby images with similar structures in the graph. We have evaluated our proposed groupwise registration method on both infant and adult data sets, by also comparing with the conventional group-mean based registration and the ABSORB methods. All experimental results show that our proposed method can achieve better performance in terms of registration accuracy and robustness.
\end{abstract}

\section{Keywords}

Unbiased groupwise registration; graph shrinking; image manifold; diffeomorphism

\footnotetext{
(C) 2013 Elsevier Inc. All rights reserved.

*Corresponding author.
}

Publisher's Disclaimer: This is a PDF file of an unedited manuscript that has been accepted for publication. As a service to our customers we are providing this early version of the manuscript. The manuscript will undergo copyediting, typesetting, and review of the resulting proof before it is published in its final citable form. Please note that during the production process errors may be discovered which could affect the content, and all legal disclaimers that apply to the journal pertain. 


\section{Introduction}

Modern imaging technology such as Magnetic Resonance Imaging (MRI) provides a safe and non-invasive way for performing clinical diagnosis and clinical research, e.g., on human brain development, aging, and disease-induced anomalies. For reliable estimation of disease related microstructure difference, it is important to develop accurate image registration methods for dealing with the possible tiny intra-subject changes in longitudinal studies and the complicated inter-subject variability in cross-sectional studies (Auzias et al. (2011); Durrleman et al. (2011); Fonov et al. (2011); Jia et al. (2011); Wu et al. (2012)).

Image registration is designed to estimate the deformation field for warping each subject image onto the standard space, thus measuring the relative difference between the subject and the template in the standard space. Although aligned images become similar after registration, their intrinsic anatomical characteristics are encoded in their deformation pathway from their individual space to the standard space. Therefore, voxel-based methods can be applied to reveal the structure/functional difference between populations by comparing the Jacobian determinants of the deformation fields. So far, many pairwise registration algorithms, based on either intensities or features, have been developed (Avants and Gee (2004); Avants et al. (2008); Beg (2003); Beg et al. (2005); Cootes et al. (2010); Fletcher and Joshi (2007); Hamm et al. (2009); Hernandez et al. (2008); Hirani et al. (2001); Holm et al. (2009); Shen and Davatzikos (2002); Trouvé and Younes (2005); Vercauteren et al. $(2009,2008)$; Younes (2007)), with the comprehensive survey provided in Klein et al. (2009) and Younes (2010). One major limitation of the pairwise registration algorithms is the requirement of selecting a particular image as a template. This could unfortunately lead to bias on the final analysis results, due to high anatomical variability across individual human brain images (Toga and Thompson (2001)).

To alleviate this limitation, groupwise registration has been recently proposed to warp all images simultaneously onto the common space (Ashburner and Friston (2009); Durrleman et al. (2011); Fletcher et al. (2009); Fonov et al. (2011); Jia et al. (2010, 2011); Joshi et al. (2004); Klein et al. (2009); Noblet et al. (2012); Wang et al. (2010); Wu et al. (2011, 2012); Xie et al. (2010)). Thus, the overall registration accuracy for all images under registration could be improved by avoiding template selection, and also the anatomical differences among images can be better delineated.

Most existing groupwise registration algorithms assume one center among a group of images under registration (Ashburner and Friston (2009); Beg and Miller (2007); Cardoso, et al. (2012); Durrleman et al. (2011); Fonov et al. (2011); Gerber et al. (2010); Lorenzen et al. (2005); Noblet et al. (2012); Ribbens et al. (2010); Sabuncu et al. (2009); Thompson et al. (2000); Vialard et al. (2011); Wang et al. (2010); Wu et al. (2012); Xie et al. (2010)). Thus, each individual image is usually warped in every iteration to the tentatively-estimated group-mean image. To overcome the bias of selecting a particular image as a template, several approach have been proposed (Dhollander et al. (2011); Fletcher et al. (2009); Joshi et al. (2004); Leemput (2009); Studholme and Cardenas (2004); Wang et al. (2005); Wu et al. (2011)). The algorithm developed by Joshi et al. in 2004 (Joshi et al. (2004)) is one of the most popular methods, where Karcher mean on manifolds (Fletcher and Joshi (2007); 
Karcher (1977)) is used to model the groupwise registration problem. However, one critical limitation of this algorithm is the use of simple averaging in estimating the group-mean image. Although no bias is introduced in the registration, it could cause the unrecoverable information loss in terms of details of anatomical structures (Wu et al. (2011)). When the images are not well registered (i.e., in the very beginning of groupwise registration), the resulted fuzzy group-mean image could seriously affect the overall accuracy in the final registration. To address this limitation, Fletcher et al. (Fletcher et al. (2009)) replaced the simple averaging with the geometric median on Riemannian manifolds. Recently, Wu et al. (Wu et al. (2011)) proposed a "Sharp-Mean" method that uses the adaptive weighing strategy to dynamically control the fuzzyness of the group-mean image throughout the whole groupwise registration procedure. The advantage of the "Sharp-Mean" method is that the anatomical structures in the group-mean image are always kept clear enough to drive all images towards the data center. On the other hand, Studholme and Cardenas (Studholme and Cardenas (2004)) normalized the brain images by introducing the joint intensity distribution of image values to form a general model of aligning the population data, while Wang et al. (Wang et al. (2005)) proposed an atlas construction method based on a viscous fluid deformation model and applied it to the segmentation of MR brain images. Leemput et al. (Leemput (2009)) generalized the probabilistic atlas model by using a mesh-based atlas representation instead of the average atlas. More recently, Dhollander et al. (Dhollander et al. (2011)) developed a novel multi-subject multi-channel diffeomorphic registration algorithm for DWI atlas construction in Q-space and Cardoso et al. (Cardoso, et al. (2012)) developed a new framework by applying the information propagation method.

Recently, more and more clinical studies and trials need analysis of large population data sets (Durrleman et al. (2011); Fonov et al. (2011)). Due to large inter-subject variations and the difficulty of using a single data center to describe the distribution of all population data, the group-mean based registration methods have their limitations in registering large population data. To address this issue, several methods have been proposed so far (Beg and Miller (2007); Ribbens et al. (2010); Sabuncu et al. (2009); Thompson et al. (2000); Wang et al. (2010)). For example, Thompson et al. (Thompson et al. (2000)) constructed a diseasespecific atlas to represent the human brain in Alzheimer's disease. This approach is perhaps the most rigorous way of measuring shape. However, their current limitation is that it relies heavily on a perfect registration between the template and the individuals. Then, Blezek and Miller (Beg and Miller (2007)) explored the atlas stratification for multiple templates estimation from a set of images by combining with the mean shift method. Recently, Sabuncu et al. (Sabuncu et al. (2009)) clustered the subject images of a population to some groups for forming subgroup-atlases and Ribbens et al. (Ribbens et al. (2010)) established a probabilistic framework for segmentation, clustering and atlas construction based on the EM algorithm. However, it should be pointed out that the variations between the centers of subgroups may be large which may increase the difficulties for registration between these centers. More recently, Wang et al. (Wang et al. (2010)) proposed a hierarchical framework to cluster images into a pyramid of classes. Then, intra-class registration is performed to register all (similar) images within each class and generate its representative center image. The center images of all classes will be further registered from the bottom to the top in the pyramid, until all images are registered. Therefore, this groupwise registration framework 
can efficiently register a large image data set with relatively better registration performance since each time only similar images need to be registered.

Inspired by this hierarchical pyramid-based method, we introduce a graph into the groupwise registration, and then formulate the procedure of agglomerating all images into the common space as the dynamic evolution of graph shrinkage. Specifically, we consider each image as a node in the graph with each edge representing the geodesic pathway between the two nodes. Thus, the objective of our method becomes to shrink the graph with its topology well preserved, until all nodes become close to each other. Given the graph, we are able to estimate velocity for deforming a certain node to its connected neighbour (i.e., by using the Log-Demons method (Vercauteren et al. (2008)). During graph shrinking, each image (or the node in the graph) is driven by the average velocities towards its connected images (or nodes in the graph), and warped along the geodesic on the image manifold. To this end, neither the group-mean image nor any individual image is required to act as, and thus bias, the center of the group during registration.

Our method is also developed partly upon our previous ABSORB method (Jia et al. (2010)) where each image is deformed locally w.r.t. its neighbors within the learned image manifold. However, compared to ABSORB, our method has several advantages. (1) Our method is able to preserve the entire distribution of images, while ABSORB is only able to maintain the local distribution. (2) ABSORB still needs to estimate a tentative group-mean image for ensuring that each individual image is warped towards the group center, not the opposite direction, while our method totally avoids the estimation of tentative group-mean image which could be ill-determined especially in the beginning. (3) ABSORB simply averages the deformations w.r.t. the neighboring images to warp each individual image, where the averaged deformation could be non-invertible. On the contrary, we address this limitation by employing diffeomorphism in our graph shrinkage procedure.

The rest of this paper is organized below. In Section II, we explain the mathematical formulations of our graph shrinkage model for groupwise registration. Then, in Section III, we comprehensively evaluate the registration performance of our proposed groupwise registration method using both longitudinal infant data set and the adult brain data set, by comparing with the conventional group-mean based method and our previous ABSORB method. Finally, the whole paper is concluded in Section IV.

\section{Methods}

\subsection{Background}

Let $\left\{I_{i}\right\}_{i=1}^{N}$ be a group of $N$ subject images to be registered. They are assumed to be sitting on the image manifold $\mathscr{M}$. In the groupwise registration method proposed by Joshi et al. (Joshi et al. (2004)), the population center is modeled as the Karcher mean $I_{c}$ under an $H^{1}$ (Sobolev) metric, as described below:

$$
I_{c}=\underset{I \in \mathscr{M}}{\operatorname{argmin}} \sum_{i=1}^{N} d^{2}\left(I_{i}, I\right)
$$


where $d(\cdot, \cdot)$ is the geodesic distance between two images on $\mathscr{W}$. For instance, $d$ is defined as an $H^{1}$ metric in (Joshi et al. (2004)) and a metamorphosis metric in (Fletcher et al. (2009)).

It is easy to find that the mean image $I_{c}$ of images $\left\{I_{i}\right\}_{i=1}^{N}$ should be a point on the manifold $\mathscr{W}$, from which the summation of lengths of geodesics to all subject images is minimal (See Fig. 1.). Eq. (1) also describes that the group-mean image is a point on the manifold and it has the close distance to all subject images.

For any pair of images $I_{i}, I_{j} \in \mathscr{W}$, their geodesic distance $d\left(I_{i}, I_{j}\right)$ is given as:

$$
d\left(I_{i}, I_{j}\right)=\int_{0}^{1}\left\|\dot{\gamma}_{i j}(s)\right\| d s=\int_{0}^{1}\left\|v_{i, j}^{s}\right\| d s, \quad \text { (2) }
$$

where $v_{i, j}^{s}$ is the velocity vector (or tangent vector in (Helgason (2001))) on the geodesic $\gamma_{i j}(s)$, with $\gamma_{i j}(0)=I_{i}$ and $\gamma_{i j}(1)=I_{j}$, and the superscript $s$ is an arc length parameter. From the definition, the geodesic on manifold is a curve parameterized with constant velocity, i.e., $\left\|v_{i, j}^{s}\right\|=\left\|v_{i, j}^{0}\right\|, \forall s \in[0,1]$. Hence, we have

$$
d\left(I_{i}, I_{j}\right)=\left\|v_{i, j}^{0}\right\|
$$

by substituting $v_{i, j}^{s}$ with $v_{i, j}^{0}$ in Eq. (2). This indicates that the length of the geodesic pathway from $I_{i}$ to $I_{j}$ eventually equals the length of the velocity vector at the initial point $I_{i}(s=0)$.

For convenience, in the following, we omit the superscript ' 0 ' in $v_{i, j}^{0}$ and use $v_{i, j}$ to represent the constant velocity vector of the geodesic from $I_{i}$ to $I_{j}$.

\subsection{Energy Function of Graph Shrinkage Model}

The goal of groupwise registration is to simultaneously register all subject images to the population center. Here we assume the deforming of each individual image as a dynamic procedure of time variable $t$. Thus, $I_{i}(t)$ can be used to represent the deformed image $I_{i}$ at time $t$. When $t$ approaches infinite (i.e., at the end of groupwise registration), the overall distance $F(\tilde{t})$ between all pairs of the deformed images should be minimal:

$$
\tilde{F}(t)=\sum_{i, j=1}^{N} d^{2}\left(I_{i}(t), I_{j}(t)\right)=\sum_{i, j=1}^{N}\left\|v_{i, j}(t)\right\|^{2},
$$

where $v_{i, j}(t)$ denotes the constant velocity vector from image $I_{i}(t)$ to image $I_{j}(t)$ at time $t$.

In general, deformable image registration is just diffeomorphic registration using a stationary velocity field. Since it is challenging to register two images with large anatomical differences, minimizing $\tilde{F(t)}$ by considering the registration of all possible pairs of images might undermine the overall registration performance. Inspired by the ABSORB method, it is reasonable to consider the registration only between the two images with similar anatomical structures. Therefore, we introduce the variable $e_{i j}$ to indicate whether images $I_{i}(t)$ and $I_{j}(t)$ are similar enough $\left(e_{i j}=1\right)$ or not $\left(e_{i j}=0\right)$. Thus, the overall distance $F(\tilde{t})$ in Eq. (4) can be rewritten as: 


$$
F(t)=\sum_{i, j=1}^{N} e_{i j}\left\|v_{i, j}(t)\right\|^{2} .
$$

Next, we use a graph defined in the brain image manifold to interpret $F(t)$ in Eq. (5). Let $I(t)=\left\{I_{i}(t)\right\}_{i=1}^{N}$ be the graph node and $E=\left\{e_{i j}: i, j=1, \ldots, N.\right\}$ be the edge between two nodes in the graph. $e_{i j}=1$ means existence of a link between $I_{i}(t)$ and $I_{j}(t)$. Otherwise, there is no direct link between $I_{i}(t)$ and $I_{j}(t)$ in the graph. Also, we define a weighted adjacency matrix $V(t)=\left(v_{i, j}(t)\right)$ to describe the similarity of two images, such that $v_{i, j}(t)>0$ if $e_{i j}=1$ and $v_{i, j}(t)=0$ otherwise. Since we do not allow self-loop in the graph, we further define $v_{i, i}(t)=0$ for all $i \in\{1, \ldots, N\}$. Then, the graph in our application can be defined as $\mathscr{G}(t)=$ $(I(t), E, V(t))$. Since $\mathscr{G}(t)$ is an undirected graph, $V(t)$ is a symmetric matrix.

The principle behind $F(t)$ in Eq. (5) is demonstrated in Fig. 2. First, all images are assumed to be sitting in a high-dimension manifold. Then, the topology of their distribution can be described by a graph, where the graph edges denote the local connectivity between graph nodes. Specifically, the velocity vector $v_{i, j}(t)$ is associated with each graph edge, where the integration along $v_{i, j}(t)$ forms the geodesic distance from $I_{i}(t)$ to $I_{j}(t)$. Thus, the minimization of $F(t)$ can be regarded as a dynamic graph shrinking procedure, which deforms each image from $I_{i}(t)$ to $I_{i}(t+\Delta t)$ with the decreased overall geodesic distance, while keeping the topology of the entire graph. As shown in Fig. 2, all the images (in blue dots) at time $t$ are the nodes in the graph where the graph edges are denoted by the red solid curves. At time $t+$ $\Delta t$, each node $I_{i}(t)$ is deformed to its next position $I_{i}(t+\Delta t)$ (in purple dots) on the manifold. The graph $\mathscr{G}(t+\Delta t)$ (in purple dots and green curves) keeps the same topology with $\mathscr{G}(t)$ (in blue dots and red curves). As time $t$ increases, all $I_{i}(t)$ s meet at the population center at the end of groupwise registration.

Therefore, the key steps in our method are (1) constructing the graph on the brain manifold and (2) deforming image $I_{i}(t)$ toward the hidden population center at time $t$. We will explain these two steps next.

\subsection{Graph Construction}

In the beginning of groupwise registration $(t=0)$, an $N \times N$ distance matrix can be calculated with each element corresponding to the geodesic distance $d\left(I_{i}(0), I_{j}(0)\right)$ between two images $I_{i}(0)$ and $I_{j}(0)$, where the geodesic distance is estimated through the LogDemons method (Vercauteren et al. (2008)). The naïve solution for graph construction is to set a threshold $h$ and then remove elements with geodesic distances higher than the threshold $h$, i.e.,

$$
e_{i j}= \begin{cases}1, & d\left(I_{i}(0), I_{j}(0)\right)<h, i \neq j \\ 0, & \text { otherwise }\end{cases}
$$

Here, we go one step further to adaptively construct the graph according to the distribution of images. Specifically, two criteria are used to construct the graph: (C1) for any two nodes 
in the graph, there should be at least one path connecting these two nodes; (C2) the number of graph edges should be as low as possible, for saving the computational cost during the groupwise registration.

Accordingly, we propose a line-search based method to determine the optimal threshold $h$. Given the geodesic distance between any two images $I_{i}(0)$ and $I_{j}(0)$, we set the search range within the low bound $b_{L}=0$ and the upper bound $b_{H}=\max _{i, j} d\left(I_{i}(0), I_{j}(0)\right)$. The optimal threshold $h$ is updated as $h=b_{L}+\lambda\left(b_{H}-b_{L}\right)$, where $\lambda \in(0,1)$ is a scalar specifying the step size in line search. Then, if the tentatively constructed graph satisfies the criterion $(\mathrm{C} 1)$, the upper bound $b_{H}$ will be decreased to $h$; otherwise, the low bound $b_{L}$ will be increased to $h$. We repeat these steps until the low bound $b_{L}$ is close to the upper bound $b_{H}$. The whole graph construction algorithm can be summarized as follows.

\section{Algorithm 1}

Graph generation algorithm

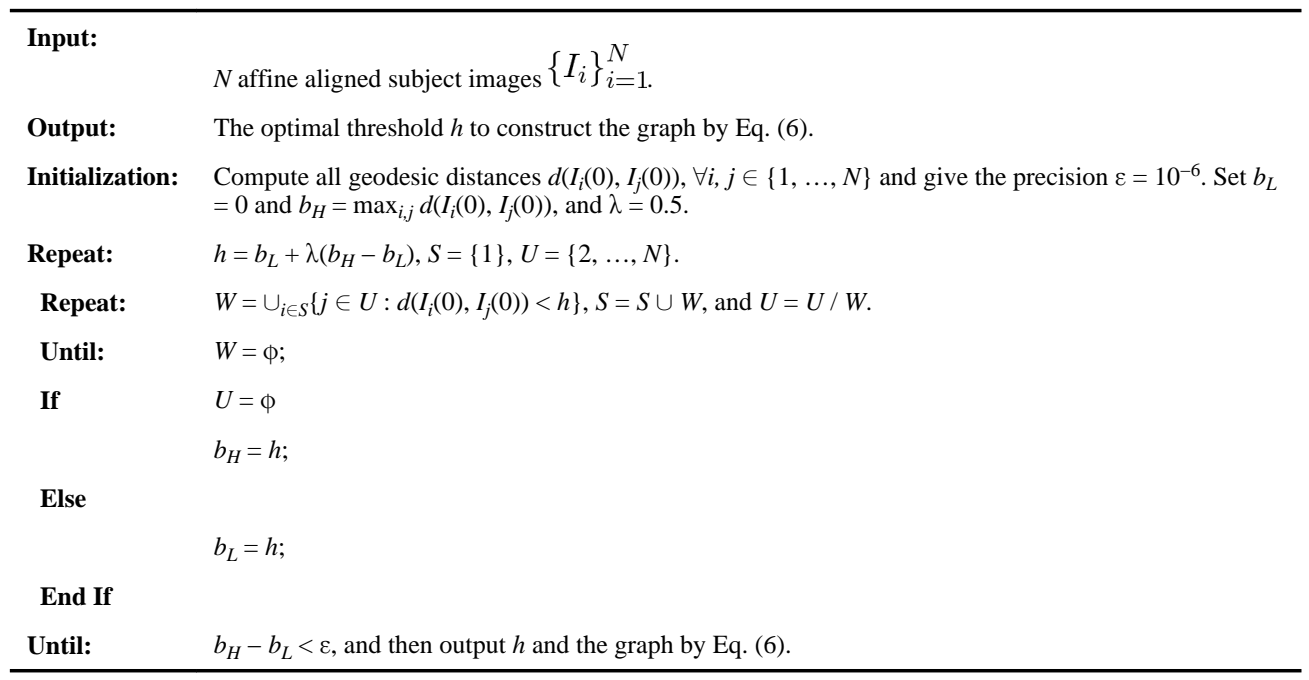

\subsection{Graph Shrinkage}

As we formulate the problem of groupwise registration as the dynamic shrinkage of graph, it is critical to determine the deformation of each image $I_{i}(t)$ at time $t$, which can minimize $F(t)$ in Eq. (5) during the graph shrinking. To solve this minimization problem, we propose a descent method as detailed below.

Suppose each image has been deformed from $I_{i}(0)$ to $I_{i}(t)$ (at time $t$ ). Similar to the ABSORB method, we can move $I_{i}(t)$ along the average velocity direction on the manifold according to its connected images in the graph $\mathscr{G}(t)$. Since the velocity vector sits on the tangent space of $I_{i}(t)$ on the manifold $\mathscr{W}$, it can be efficiently calculated by linear averaging as below:

$$
\hat{v}_{i}(t)=\frac{1}{N_{i}} \sum_{j=1}^{N} e_{i j} v_{i, j}(t),
$$


where $N_{i}=\sum_{j=1}^{N} e_{i j}$ is the number of connections for $I_{i}(t)$. It is worth noting that we use Log-Demons (Vercauteren et al. (2008)) to estimate the velocity vector $v_{i, j}(t)$ between $I_{i}(t)$ and $I_{j}(t)$. The geodesic from $I_{i}(t)$ to $I_{j}(t)$ can be calculated by $\exp \left(v_{i, j}(t)\right)$.

Given a time increment $\Delta t$, image $I_{i}(t)$ is deformed to $I_{i}(t+\Delta t)$ along the geodesic $\exp \left(v_{i}(t)\right.$. $\Delta t)$, where the velocity direction is steered by $\hat{v_{i}(t)}(\mathrm{Eq} .(7))$ and the step size is determined by $\Delta t$. We will explain the calculation of optimal $\Delta t$ later. As shown in Fig. 3, the geodesics from $I_{i}(t)$ to $I_{i}(t+\Delta t), I_{i}(t+\Delta t)$ to $I_{j}(t+\Delta t), I_{j}(t+\Delta t)$ to $I_{j}(t)$, and $I_{j}(t)$ to $I_{i}(t)$ form a closed loop, given as:

$$
\exp \left(v_{i, j}(t+\Delta t)\right)=\exp \left(-\hat{v}_{i}(t) \cdot \Delta t\right) \exp \left(v_{i, j}(t)\right) \circ \exp \left(\hat{v}_{j}(t) \cdot \Delta t\right)
$$

where 'o' denotes the composition of two geodesics. If $\Delta t$ is small enough, the velocity vector $v_{i, j}(t+\Delta t)$ can be approximated by applying Baker-Campbell-Hausdorff formula (Helgason (2001)) on Eq. (8) as:

$$
v_{i, j}(t+\Delta t)=-\hat{v}_{i}(t) \cdot \Delta t+v_{i, j}(t)+\tau_{j i}\left(\hat{v}_{j}(t)\right) \cdot \Delta t+o\left(\hat{v}_{i}(t) \cdot \Delta t\right),
$$

where $\tau_{j i}: T_{I_{j}} \mathscr{M} 7 \mapsto T_{I_{j}} \mathscr{M}$ denotes the linearization of the left translation from $I_{j}(t)$ to $I_{i}(t)$, as shown in Fig. 3. Thanks to the left-invariant structure of the diffeomorphism group, we have the left invariant metric on this manifold. That is, $\left\langle\tau_{j i}\left(\hat{v_{j}} \hat{(t)}\right), \tau_{j i}\left(\hat{\left.v_{j}(t)\right)}\right\rangle=\left\langle\hat{v_{j}(t),} \hat{\left.v_{j}(t)\right\rangle}\right.\right.$. For more details of the diffeomorphism groups of compact manifolds, we refer to (Smolentsev (2007)).

Then, we have the following convergence theorem affirming that $F(t)$ is a monotonically decreasing function of time $t$ along the velocity direction defined in Eq. (7).

Theorem 1. The velocity fields defined in (7) make the objective function $F(t)$ strictly and monotonically decreasing.

Proof: We directly calculate the derivative of the objective function (5) with respect to any fixed time variable $t$ by

$$
F^{\prime}(t)=\lim _{\Delta t \rightarrow 0} \frac{\Delta F(t ; \Delta t)}{\Delta t}
$$

where

$$
\Delta F(t ; \Delta t)=F(t+\Delta t)-F(t)=\sum_{i, j=1}^{N} e_{i j}\left(\left\|v_{i, j}(t+\Delta t)\right\|^{2}-\left\|v_{i, j}(t)\right\|^{2}\right)=\sum_{i, j=1}^{N} e_{i j}\left(\left\langle v_{i, j}(t+\Delta t), v_{i, j}(t+\Delta t)\right\rangle-\left\langle v_{i, j}(t), v_{i, j}(t)\right\rangle\right)
$$

Substituting Eq. (9) to above equation, we have 


$$
\Delta F(t ; \Delta t)=-2 \Delta t \underbrace{\sum_{i, j=1}^{N} e_{i j}\left\langle v_{i, j}(t), \hat{v}_{i}(t)-\tau_{j i}\left(\hat{v}_{j}(t)\right)\right\rangle}_{(\mathrm{I})}+\Delta t^{2} \underbrace{\sum_{i, j=1}^{N} e_{i j}\left\langle\hat{v}_{i}(t)-\tau_{j i}\left(\hat{v}_{j}(t)\right), \hat{v}_{i}(t)-\tau_{j i}\left(\hat{v}_{j}(t)\right)\right\rangle}_{(\mathrm{II})}+o\left(\Delta t^{2}\left\|\hat{v}_{i}(t)\right\|^{2}\right)
$$

where terms (I) and (II) are calculated as follows.

$$
\begin{aligned}
(\mathrm{I})= & \sum_{i, j=1}^{N} e_{i j}\left\langle v_{i, j}(t), \hat{v}_{i}(t)\right\rangle \\
& -\sum_{i, j=1}^{N} e_{i j}\left\langle v_{i, j}(t), \tau_{j i}\left(\hat{v}_{j}(t)\right)\right\rangle \\
& =\sum_{i=1}^{N}\left\langle\sum_{j=1}^{N} e_{i j} v_{i, j}(t), \hat{v}_{i}(t)\right\rangle \\
& -\sum_{j=1}^{N}\left\langle\sum_{i=1}^{N} e_{i j} \tau_{i j}\left(v_{i, j}(t)\right), \hat{v}_{j}(t)\right\rangle \\
= & \sum_{i=1}^{N} N_{i}\left\|\hat{v}_{i}(t)\right\|^{2} \\
& +\sum_{j=1}^{N}\left\langle\sum_{i=1}^{N} e_{j i} v_{j, i}(t), \hat{v}_{j}(t)\right\rangle \\
= & 2 \sum_{i=1}^{N} N_{i}\left\|\hat{v}_{i}(t)\right\|^{2} .
\end{aligned}
$$

and

$$
\begin{aligned}
(\mathrm{II}) & =\sum_{i, j=1}^{N} e_{i j}\left\|\hat{v}_{i}(t)\right\|^{2} \\
& +\sum_{i, j=1}^{N} e_{i j}\left\|\hat{v}_{j}(t)\right\|^{2} \\
& -2 \sum_{i, j=1}^{N} e_{i j}\left\langle\hat{v}_{i}(t), \tau_{j i}\left(\hat{v}_{j}(t)\right)\right\rangle=2 \sum_{i=1}^{N} N_{i}\left\|\hat{v}_{i}(t)\right\|^{2}-2 \sum_{i=1}^{N}\left\langle\hat{v}_{i}(t), \sum_{j=1}^{N} e_{i j} \tau_{j i}\left(\hat{v}_{j}(t)\right)\right\rangle \\
= & 2 \sum_{i=1}^{N}\left(N_{i}\right. \\
& +1)\left\|\hat{v}_{i}(t)\right\|^{2} .
\end{aligned}
$$

where the last equation holds because $\hat{v}_{i}(t)=-\sum_{i=1}^{N} e_{i j} \tau_{j i}\left(\hat{v}_{j}(t)\right)$.

Therefore, we have 


$$
\Delta F(t ; \Delta t)=-4\left(\sum_{i=1}^{N} N_{i}\left\|\hat{v}_{i}(t)\right\|^{2}\right) \cdot \Delta t+2\left(\sum_{i=1}^{N}\left(N_{i}+1\right)\left\|\hat{v}_{i}(t)\right\|^{2}\right) \cdot \Delta t^{2}+o\left(\Delta t^{2}\left\|\hat{v}_{i}(t)\right\|^{2}\right) .
$$

Then, $F^{\prime}(t)=-4\left(\sum_{i=1}^{N} N_{i}\left\|\hat{v}_{i}(t)\right\|^{2}\right)$ is always negative for all $t>0$. Therefore, the objective function (5) is always strictly and monotonically decreasing and then there is the limit at the end of the groupwise registration because every bounded monotonic sequence has a limit.

\subsection{Numerical Implementation}

By Theorem 1, the velocity vector defined by Eq. (7) makes the objective function (5) decreasing at any time $t$. Then, to implement this minimization procedure, we should design an iterative process. For any step $k, t^{k}$ is used to present the current time. First, we calculate the velocity vectors $\hat{v}_{i}^{k}:=\hat{v}_{i}\left(t^{k}\right),(i=1, \ldots, N)$ by Eq. (7). Then, along these velocity vectors, the stepsize $\Delta t^{k}$ should be determined. According to the convergent condition of the Taylor series of exponential map which makes the approximation (9) held, there should be $\Delta t^{k} \cdot\left\|\hat{v}_{i}^{k}\right\|<1$ for all $i=1, \ldots, N$, and therefore $\Delta t^{k}<1 / \max _{i}\left\|\hat{v}_{i}^{k}\right\|$. Under this condition, the increment $\Delta F\left(t^{k} ; \Delta t\right):=\sum_{i, j}^{N}=1 e_{i, j}\left(\| v_{i, j}\left(t^{k}+\Delta t\left\|^{2}-\right\| v_{i, j}\left(t^{k}\right) \|^{2}\right)\right.$ can be approximated by a positive definite quadratic function (10) of $\Delta t$. Then, to accelerate the algorithm, $\Delta t^{k}$ should be selected to make the increment $\Delta F\left(t^{k} ; \Delta t\right)$ decreased as large as possible. That is, at each step, the objective function is sufficiently decreased. Thus, it is straightforward to determine the optimal value of $\Delta t$ by

$$
\Delta t^{k}=\frac{\sum_{i=1}^{N} N_{i}\left\|\hat{v}_{i}^{k}\right\|^{2}}{\sum_{i=1}^{N}\left(N_{i}+1\right)\left\|\hat{v}_{i}^{k}\right\|^{2}}
$$

Especially, when the graph is fully connected, i.e. $N_{i} \equiv N-1, i=1, \ldots, N$, we have

$$
\Delta t^{k} \equiv \frac{N-1}{N}
$$

This is consistent with Theorem 3.1 in (Noblet et al. (2012)) which deforms one image w.r.t. all other images.

In general, the $k^{\text {th }}$ stepsize $\Delta t^{k}$ is selected by

$$
\Delta t^{k}=\min \left\{\frac{1}{\max _{i}\left\|\hat{v}_{i}^{k}\right\|}, \frac{\sum_{i=1}^{N} N_{i}\left\|\hat{v}_{i}^{k}\right\|^{2}}{\sum_{i=1}^{N}\left(N_{i}+1\right)\left\|\hat{v}_{i}^{k}\right\|^{2}}\right\} .
$$




\subsection{Summary}

In our groupwise registration method, we first use a graph to model the distribution of all images on the manifold. Then, the groupwise registration is formulated as the graph shrinking procedure, where each image in the graph deforms along the graph edge on the manifold. Our whole method is summarized below.

\section{Algorithm 2}

Graph Shrinking Algorithm

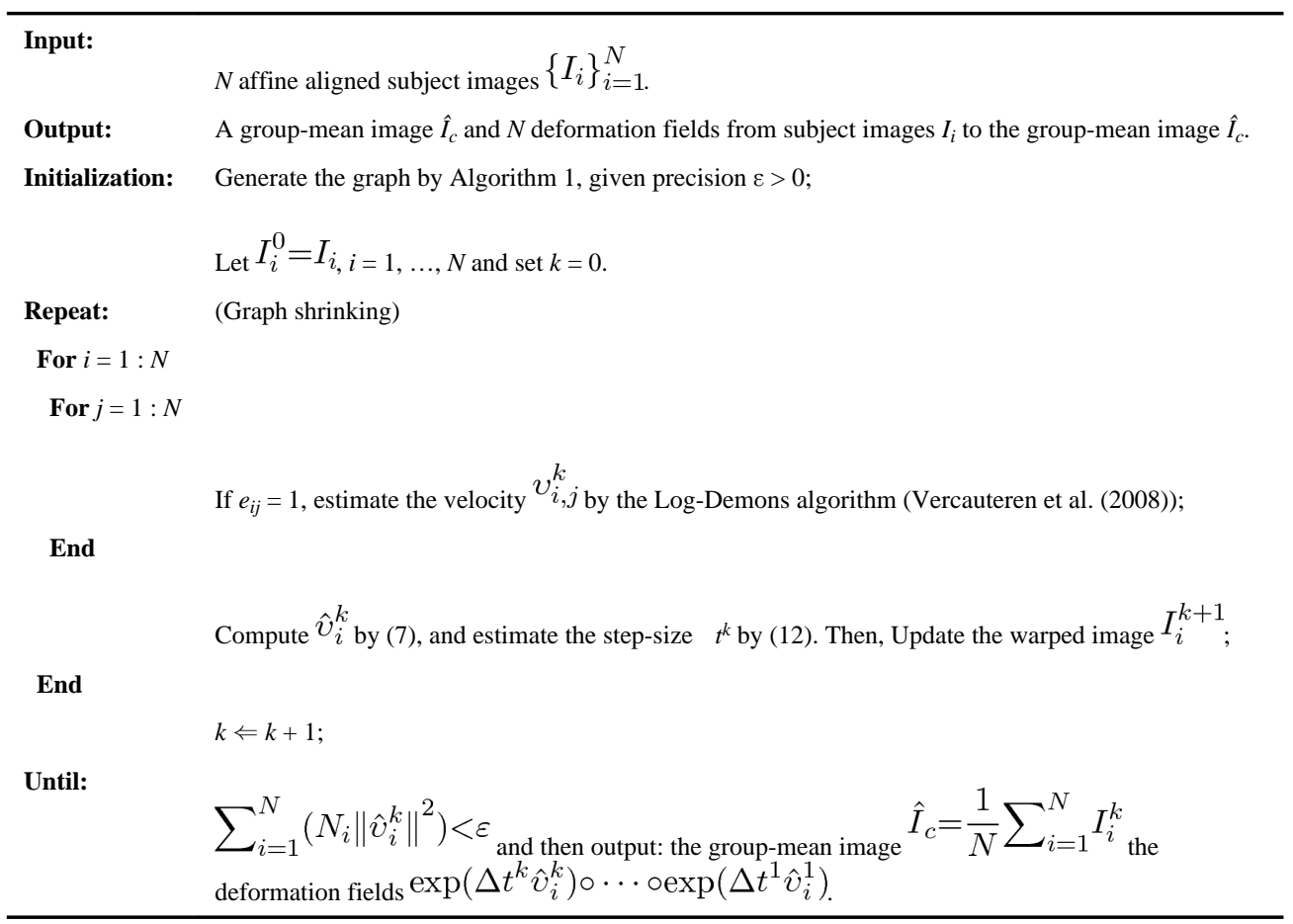

\section{Experimental Results}

In this section, we evaluate the registration performance of our proposed groupwise registration method on a synthetic dataset with $612 \mathrm{D}$ images, and two real data sets of human brain images, in which the first data set consists of longitudinal infant subjects, each with MR images scanned at 0, 3, 6, 9, 12 and 18 months of ages, respectively and the second data set includes 30 adult brain images, each with 83 manually labelled ROIs. In the first experiment, we portray the registration paths of all images on the image manifold. Furthermore, for comparison in real data sets, we select the widely used group-mean registration method and our previous ABSORB method. To quantitatively evaluate the registration accuracy, we use the Dice ratio to measure the overlap degree between ROI A and ROI B, given as:

$$
\operatorname{Dice}(A, B)=2 \times \frac{|A \cap B|}{|A|+|B|},
$$


where $|\cdot|$ means the volume of the particular ROI. It is worth noticing that the image similarity and the Dice ratio (a kind of tissue label overlap scores) are two standards to measure the registration accuracy. Nevertheless, as mentioned by Rohifing in (Rohlfing (2010)), any kind of measures can not be fully used to describe the registration accuracy. Therefore, to better measure the accuracy of registration, these two measures are used at the same time. That is, we assume that a better registration should have a better registered similarity as well as a higher Dice ratio of all tissues, which was also used to validate 14 non-rigid registration methods in (Klein et al. (2009)).

Since no template image is selected as a reference for groupwise registration, we will construct a label image in the common space by majority voting on ROIs of all registered images, in order to use the Dice ratio to evaluate the registration performance. Specifically, the Dice ratio of each ROI will be computed between the (constructed) label image and each registered individual image in the common space. Above experiments are performed on Dell workstation (with 8 Xeon CPU@ 2.66 GHz and 32 G DDR memory).

\subsection{Groupwise Registration on Data of a Synthetic Dataset}

In this part, we first simulate the synthetic dataset that contains 61 2D images (sized 256x 256) as follows. From the baseline image (the left image in Fig. 4), three different subsets of images are generated with each subset having 20 images, respectively. These three subsets have unique evolving patterns of folds. All images shown in the right part of Fig. 4 illustrate the 1,2 , and 3 folds in individual subsets. There are thus $61(20 \times 3+1)$ images in the dataset. We project all images to a 2-dimensional space by performing PCA (Principal Component Analysis), and the distribution of images is shown in Fig. 5(a) where the solid blue circle indicates the baseline image, and the blue circles indicate the synthetic images, respectively. It is worth noting that the projected 2D PCA plane consists of the first two principal eigenvectors only.

By using the conventional group-mean method, the registration result is shown in Fig. 5(b). It is found that some images are warped out of the banded areas. By using our proposed method, the registration processes and result are portrayed in Fig. 5(c)-(e) and (f), respectively. It can be found that all images are warped almost along their respective components.

\subsection{Groupwise Registration on Data of Longitudinal Infant Brains}

The infant data used in this paper is a part of a large ongoing study of early brain development in normal children in the University of North Carolina at Chapel Hill (UNC$\mathrm{CH})$. The experimental protocols were approved by the institutional review board of the School of Medicine, UNC-CH. The parents were recruited from the UNC-CH hospitals, and written informed consent forms were obtained. T1-weighted MR brain images were collected by using a $3 \mathrm{~T}$ Siemens scanner. 160 sagittal slices were obtained with parameters: $\mathrm{TR}=1900 \mathrm{~ms}, \mathrm{TE}=4.38 \mathrm{~ms}$, Flip Angle $=7^{\circ}$. Data with motion artifacts was discarded and a rescan was made when possible. Totally 7 longitudinal infant brains are used in this experiment, each with 6 time points $(0,3,6,9,12$ and 18 months of age). The image size is 
$256 \times 256 \times 198$ and the voxel resolution is $1 \times 1 \times 1 \mathrm{~mm}^{3}$. All of these $42(6 \times 7) \mathrm{MR}$ images are displayed in Fig. 6.

We first affinely aligned all images to the first image and then use different groupwise registration methods to register them. The 3D renderings of the average images of all registered infant brain images by the conventional group-mean method, ABSORB, and our groupwise registration method are shown in Fig. 7 after about 2.1h, 6.2h, and 17h, respectively. Here, to accelerate the pairwise registration processes, all affinely aligned images have been reduced from $256 \times 256 \times 198$ to $128 \times 128 \times 100$ before groupwise registration. Note that the reduction crops image background only. All reported time costs are evaluated upon the cropped images. Furthermore, we display the multiple cross-sectional views of the average images by three registration methods in Fig. 8. Due to large anatomical variations and particularly the dynamic intensity changes as shown in Fig. 4, it is difficult, especially in the beginning of groupwise registration, to find a representative group-mean image that well describes all infant brains. Therefore, the conventional group-mean method failed to align infant brains onto the common space, as indicated by very fuzzy average image shown in Fig. 7(a) and Fig. 8(a). Both ABSORB and our groupwise registration methods achieve more reasonable registration results than the convention group-mean method, since these two methods take the advantages of detected data manifold and consider registering only the similar images during the iterative registration procedure. On the other hand, by comparing the groupwise registration results of ABSORB in Fig. 7(b) and Fig. 8(b) against the results of our method in Fig. 7(c) and Fig. 8(c), our method outperforms ABSORB in terms of the sharpness of average image and potentially the accuracy in registering each individual image.

To evaluate the overlaps of all tissues, we first segment this dataset by software "LABEL" (Shi et al. (2012)), where each brain is segmented to gray matter (GM), white matter (WM), and cerebrospinal fluid (CSF). For further details, we refer to (Shi et al. (2012)). Then, the Dice ratios on GM,WM and CSF by the conventional group-mean method, ABSORB, and our method are computed and shown in Table 1. It is easy to find that our method achieves the highest Dice ratio compared to the other two groupwise registration methods, with overall $2.85 \%$ and $2.14 \%$ improvements, respectively. Moreover, the iterative evolution of the Dice ratio on GM, WM, and CSF during the groupwise registration is shown in Fig. 9, with green, blue and red curves representing the results by the conventional group-mean method, ABSORB, and our method, respectively. It can be observed that, as the number of iterations increases, the Dice ratios on all tissue types increase much more consistently in our method than in ABSORB method. The main reason is that ABSORB does not model the entire distribution of all images as we indicated before. Instead, in ABSORB, each individual image only considers its neighboring similar images when deforming to the estimated population center, which is not sufficient to preserve the topology of the entire image distribution during the groupwise registration. Therefore, it is difficult to guarantee that all the tentatively deformed images converge to the population center, which also explains why the curves of the Dice ratio by ABSORB is not smooth as shown in Fig. 9. 


\subsection{Groupwise Registration on IXI Data set}

Here, we evaluate the registration performance of our groupwise registration method on adult brain images. IXI data set has collected nearly $600 \mathrm{MR}$ images from normal, healthy subjects, which can be downloaded at http://biomedic.doc.ic.ac.uk/brain-development/ index.php?n=Main. Datasets. 30 MR images (from 20 to 54 years old) are used in the following experiment, each with 83 manually delineated ROIs. The image size and voxel spacing are $256 \times 256 \times 198$ and $1 \times 1 \times 1 \mathrm{~mm}^{3}$, respectively. 12 typical images from IXI data set are shown in Fig. 10.

After affinely aligning all images to the first image, we separately use the conventional group-mean method, ABSORB, and our groupwise registration method to register 30 images from IXI data set. Fig. 11 and Fig. 12 show the 3D rendering and multiple cross-sectional views of the mean images by three registration methods after running about $18 \mathrm{~h}, 56 \mathrm{~h}$, and $70 \mathrm{~h}$, respectively. By visual inspection, the mean image by our groupwise registration method is much sharper than that by the conventional group-mean method or by ABSORB, indicating better registration accuracy by our method. To further show it, the map of intensity difference between all warped images and the mean image is provided in Fig. 13.

Next, as mentioned, we first construct the reference label image in the common space, based on the registered images by each registration method. Then, we calculate the Dice ratio for each of 83 ROIs between the (constructed) reference label image and each registered image. After affine alignment, the overall Dice ratio is $71.85 \%$. The Dice ratio of each ROI by the conventional group-mean method, ABSORB, and our groupwise registration method are shown in Fig. 14, in blue, red, and green, respectively. The overall Dice ratio is $74.30 \%$ by the conventional group-mean method, $75.30 \%$ by ABSORB, and $77.83 \%$ by our groupwise registration method. The evolutionary curves of average Dice ratios and their respective standard deviations of 83 ROIs are shown in Fig. 15. It is easy to find that our method achieves the best registration performance.

\section{Conclusion}

In this paper, we have developed a novel unbiased groupwise registration by first proposing a novel concept of modeling the entire image distribution as a graph. Then, the procedure of groupwise registration is formulated as the dynamic shrinkage of graph on the manifold, which brings the advantage of preserving the topology of the image distribution during the groupwise registration. Our proposed method has been evaluated on a longitudinal infant brain dataset and an adult brain dataset, where our method achieves the best registration result in comparison with the group-mean method and ABSORB. But our method is slower than the traditional methods because more pairwise registrations should be done in each iteration. Therefore, our future work includes (1) improving the speed of our method to fit the requirement of larger population data sets; (2) incorporating the longitudinal priors in our graph model for longitudinal image registration; (3) releasing the software of our groupwise registration method with user-friendly GUI to the research community. 


\section{Acknowledgments}

The authors would like to thank the editor and the anonymous reviewers for their helpful comments and suggestions. The research is supported partly by NIH grants EB006733, EB008374, EB009634, and AG041721, National Natural Science Foundation of China No. 61005002 and 11101260, Ph.D. Programs Foundation ofMinistry of Education of China No. 20103108120001, and Discpline Project at the Corresponding Level of Shanghai (A.13-0101-12-005).

\section{References}

Ashburner J, Friston K. Computing average shaped tissue probabilty templates. NeuroImage. 2009; 45(2):333-341. [PubMed: 19146961]

Auzias G, Colliot O, Glaunès J, Perrot M, Mangin J, Trouvé A, Baillet S. Diffeomorphic brain registration under exhaustive sulcal constraints. IEEE Trans. Med. Ima. 2011; 30:1214-1227.

Avants B, Gee J. Geodesic estimation for large deformation anatomical shape averaging and interpolation. NeuroImage. 2004; 23(S1):S139-S150. [PubMed: 15501083]

Avants B, Epsteinb C, Grossmanc M, Gee J. Symmetric diffeomorphic image registration with crosscorrelation: evaluating automated labeling of elderly and neurodegenerative brain. Med. Ima. Anal. 2008; 12(1):26-41.

Beg, M. Ph.D thesis. The Johns Hopkins Univ.; 2003. Variational and Computational Methods for Flows of Diffeomorphisms in Image Matching and Growth in Computational Anatomy. 2003.

Beg M, Miller M, Trouvé A, Younes L. Computing large deformation metric mappings via geodesic flows of diffeomorphisms. Int. J. Comput. Vis. 2005; 61(2):139-157.

Blezek D, Miller J. Atlas stratification. Med. Ima. Anal. 2007; 11(5):443-457.

Cardoso M, Wolz R, Modat M, Fox N, Rueckert D, Ourselin S. Geodesic information flows. MICCAI. 2012:262-270. [PubMed: 23286057]

Cootes T, Twining C, Petrovic V, Babalola K, Taylor C. Computing accurate correspondences across groups of images. IEEE Trans. Pattern Aanl. Mach. Intell. 32(11):1994-2005.

Dhollander T, Veraart J, Hecke W, Maes F, Sunaert S, Sijbers J, Suetens P. Feasibility and advantages of diffusion weighted imaging atlas construction in Q-space. MICCAI. 2011:166-173. [PubMed: 21995026]

Durrleman S, Fillard P, Pennec X, Trouvé A, Ayache N. Registration, atlas estimation and variability analysis of white matter fiber bundles modeled as currents. NeuroImage. 2011; 55(3):1073-1090. [PubMed: 21126594]

Fletcher P, Joshi S. Riemannian geometry for the statistical analysis of diffusion tensor data. Signal Process. 2007; 87:250-262.

Fletcher P, Venkatasubramanian S, Joshi S. The geometric median on Riemannian manifolds with application to robust atlas estimation. NeuroImage. 2009; 45(1-S1):S143-S152. [PubMed: 19056498]

Fonov V, Evans A, Botteron K, Almili C, McKinstry R, Collins D. the Brain Development Cooperative Group, 2011. Unbiased average age-appropriate atlases for pediatric studies. NeuroImage. 2011; 54(1):313-327. [PubMed: 20656036]

Gerber S, Tasdizen T, Fletcher P, Joshi S, Whitaker R. ADNI. Manifold modeling for brain population analysis. Med. Ima. Anal. 2010; 14:643-653.

Hamm, J.; Davatzikos, C.; Verma, R. MICCAI. London, UK: 2009. Efficient large deformation registration via geodesics on a learned manifold of images; p. 680-687.

Helgason, S. Differential Geometry, Lie Groups and Symmetric Space. Academic Press; 2001.

Hernandez, M.; Olmos, S.; Pennec, X. MICCAI. New York, USA: 2008. Comparing algorithms for diffeomorphic registration: Stationary LDDMM and Diffeomorphic Demons; p. 24-35.

Hirani, A.; Marsden, J.; Arvo, J. EMMCVPR. France: Sophia Antipolis; 2001. Averaged template matching equations; p. 528-543.

Holm D, Trouvé A, Younes L. The Euler-Poincar'e theory of metamorphosis. Quart. Appl. Math. 2009; 67:661-685. 
Jia H, Wu G, Wang Q, Shen D. ABSORB: atlas building by self-organized registration and bundling. NeuroImage. 2010; 51(3):1057-1070. [PubMed: 20226255]

Jia H, Yap P, Wu G, Wang Q, Shen D. Intermediate templates guided groupwise registration of diffusion tensor images. NeuroImage. 2011; 54(2):928-939. [PubMed: 20851197]

Joshi S, Davis B, Jomier M, Gerig G. Unbiased diffeomorphic atlas construction for computational anatomy. NeuroImage. 2004; 23(S1):S151-S160. [PubMed: 15501084]

Kacher H. Riemannian center of mass and mollifier smoothing. Commun. Pur. Appl. Math. 1977; 30:509-541.

Klein A, Sndersson J, Ardekani B, et al. Evalution of 14 nonlinear deformation algorithms applied to human brain MRI registration. NeuroImage. 2009; 46(3):786-802. [PubMed: 19195496]

Leemput K. Encoding probabilistic brain atlases using bayesian inference. IEEE Trans. Med. Ima. 2009; 28:822-837.

Lorenzen, P.; Davis, B.; Joshi, S. MICCAI. USA: Palm Springs; 2005. Unbiased atlas formation via large deformations metric mapping; p. 411-418.

Noblet V, Heinrich C, Heitz F, Armspach J. An efficient incremental strategy for constrained groupwise registration based on symmetric pairwise registration. Pattern Recog. Lett. 2012; 33:283-290.

Ribbens A, Hermans J, Maes F, Vandermeulen D, Suetens P. SPARC: unified framework for automatic segmentation, probabilistic atlas construction, registration and clustering of brain MR images. IEEE Int. Sym. Biomed. Imag. (ISBI). 2010:856-859.

Rohlfing T. Image similarity and tissue overlaps as surrogates for image registration accuracy: widely used but unreliable. IEEE Trans. Medi. Ima. 2012; 31:153-163.

Sabuncu M, Balci S, Shenton M, Golland P. Image-driven population analysis through mixture modeling. IEEE Trans. Med. Ima. 2009; 28:1473-1487.

Shen D, Davatzikos C. HAMMER: hierarchical attribute matching mechanism for elastic registration. IEEE Trans. Med. Ima. 2002; 21:1421-1439.

Shi F, Wang L, Dai Y, Gilmore J, Lin W, Shen D. LABEL: Pediatric brain extraction using learningbased meta-algorithm. NeuroImage. 2012; 62(3):1975-1986. [PubMed: 22634859]

Smolentsev N. Diffeomorphism groups of compact manifolds. J. Math. Sci. 2007; 146:6213-6312.

Studholme C, Cardenas V. A template free approach to volumetric spatial normalization of brain anatomy. Pattern Recog. Lett. 2004; 25:1191-1202.

Toga A, Thompson P. The role of image registration in brain mapping. Ima. Vis. Comput. 2001; 19(12):3-24.

Thompson P, Wood R, Mega M, Toga A. Mathematical/Computational challenges in creating deformable and probabilistic atlases of the human brain. Human Brain Mapping. 2000; 9:81-92. [PubMed: 10680765]

Trouvé A, Younes L. Metamorphoses through Lie group action. Found. Comput. Math. 2005; 5(2): 173-198.

Vercauteren, T.; Pennec, X.; Perchant, A.; Ayache, N. MICCAI. New York, USA: 2008. Symmetric Log-Domain Diffeomorphic registration: a Demons-based approach; p. 754-761.

Vercauteren T, Pennec X, Perchant A, Ayache N. Diffeomorphic demons: efficient non-parametric image registration. NeuroImage. 2009; 45(S1):S61-S72. [PubMed: 19041946]

Vialard, F.; Risser, L.; Holm, D.; Rueckert, D. Med. Ima. Understand. Anal. London: 2011. Diffeomorphic atlas estimation using Kächer mean and geodesic shooting on volumetric images.

Wang Q, Chen L, Yap P, Wu G, Shen D. Groupwise registration based on hierarchical image clustering and atlas synthesis. Human Brain Mapping. 2010; 31:1128-1140. [PubMed: 20063349]

Wang Q, Seghers D, D'Agostino E, Maes F, Vandermeulen D, Suetens P, Hammers A. Construction and validation of mean shape atlas templates for atlas-based brain image segmentation. LNCS. 2005; 3565:689-700.

Wu G, Jia H, Wang Q, Shen D. SharpMean: groupwise registration guided by sharp mean image and tree-based registration. NeuroImage. 2011; 56(4):1968-1981. [PubMed: 21440646]

Wu G, Wang Q, et al. Registration of longitudinal brain image sequences with implicit template and spatial-temporal heuristics. NeuroImage. 2012; 59(1):404-421. [PubMed: 21820065] 
Xie, Y.; Ho, J.; Vemuri, B. IEEE Conf. Comput. Vis. Pattern Recog. (CVPR). San Francisco, USA: 2010. Image atlas construction via intrinsic averaging on the manifold of images; p. 2933-2939. Younes L. Jacobi fields in groups of diffeomorphisms and applications. Quart. Appl. Math. 2007; 65:113-134.

Younes, L. Shapes and Diffeomorphisms, AMS 171. New York: Springer-Verlag; 2010. 


\section{Highlights}

- A graph on the image manifold is used to describe the entire distribution of images.

- The groupwise registration is formulated as a dynamic procedure of graph shrinkage.

- The topology of image distraction is always well maintained during groupwise registration. 


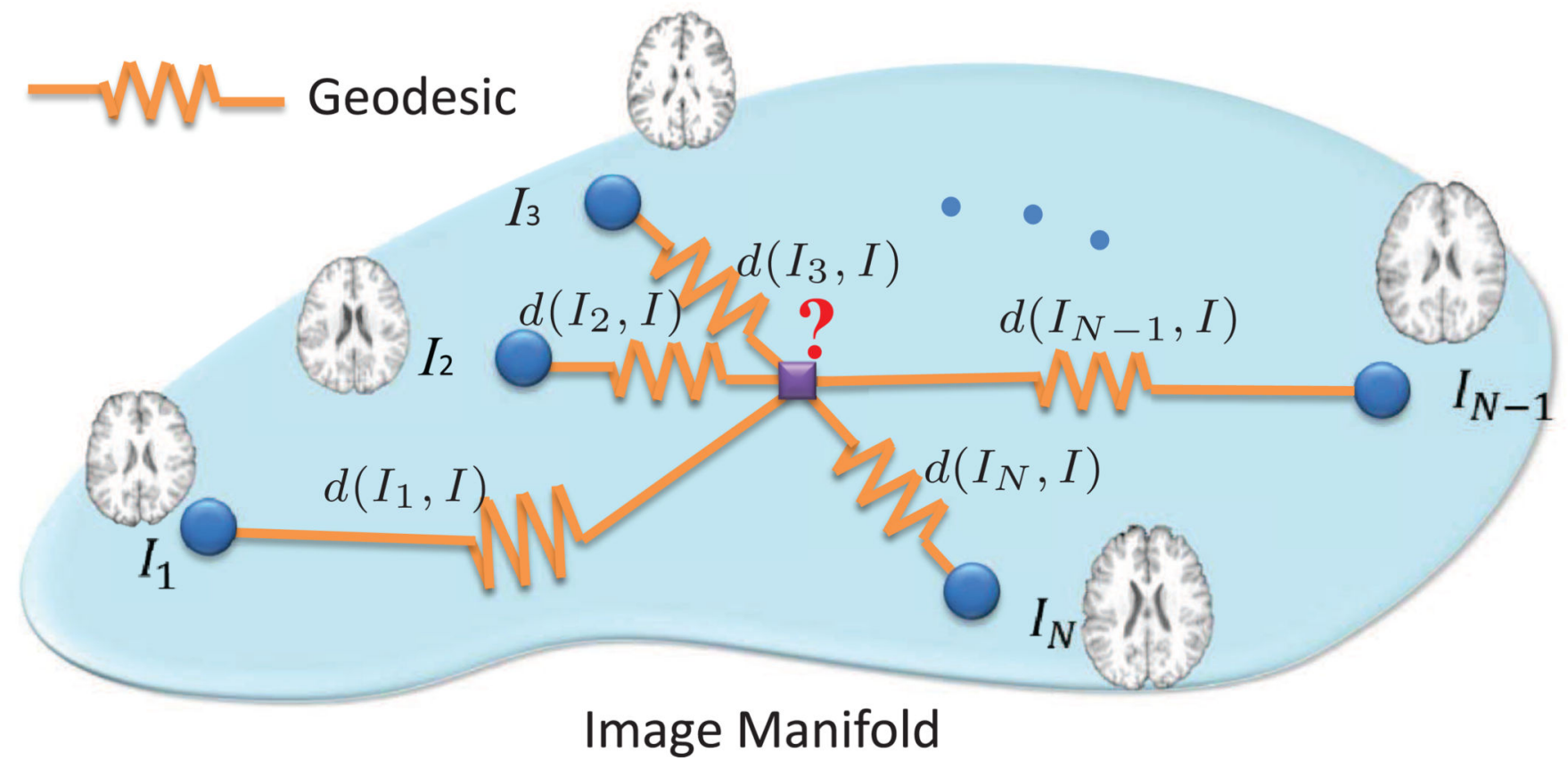

Figure 1.

Karcher mean of $N$ images on the image manifold. 
$=\Rightarrow$ geodesic from $I_{i}(t)$ to $I_{i}(t+\Delta t)$ image at $t$

$\bigcirc$ image at $t+\Delta t$

- edge at $t$

- edge at $t+\Delta t$

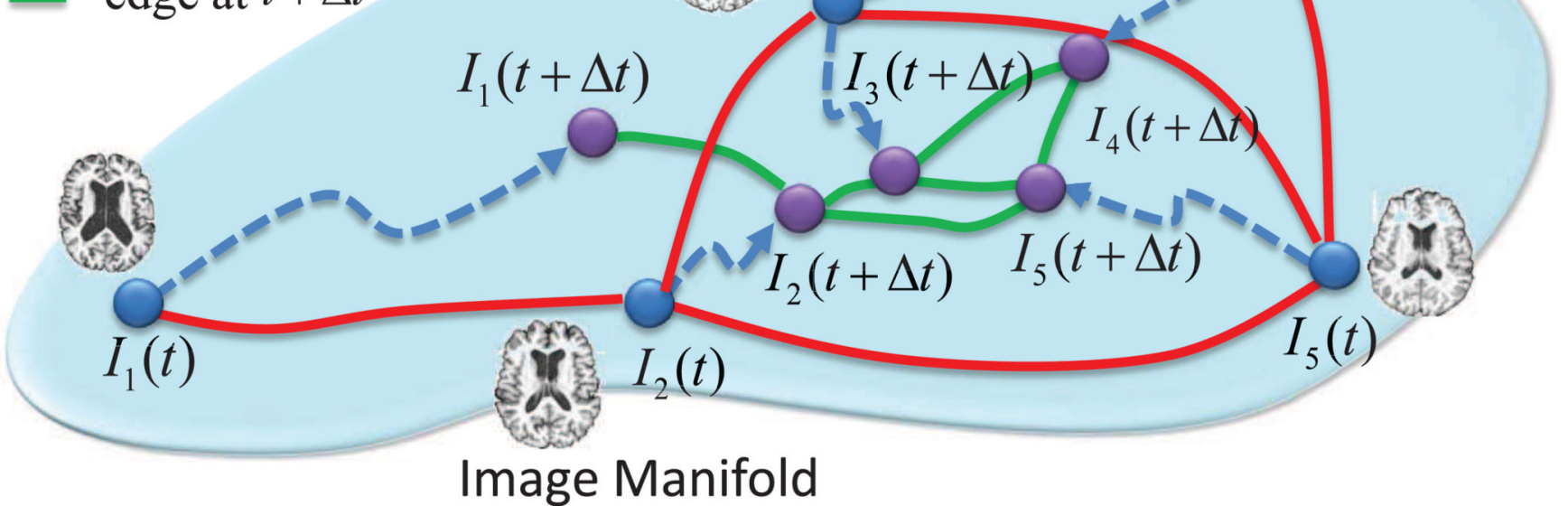

Figure 2.

Demonstration of our proposed groupwise registration by graph shrinking. 


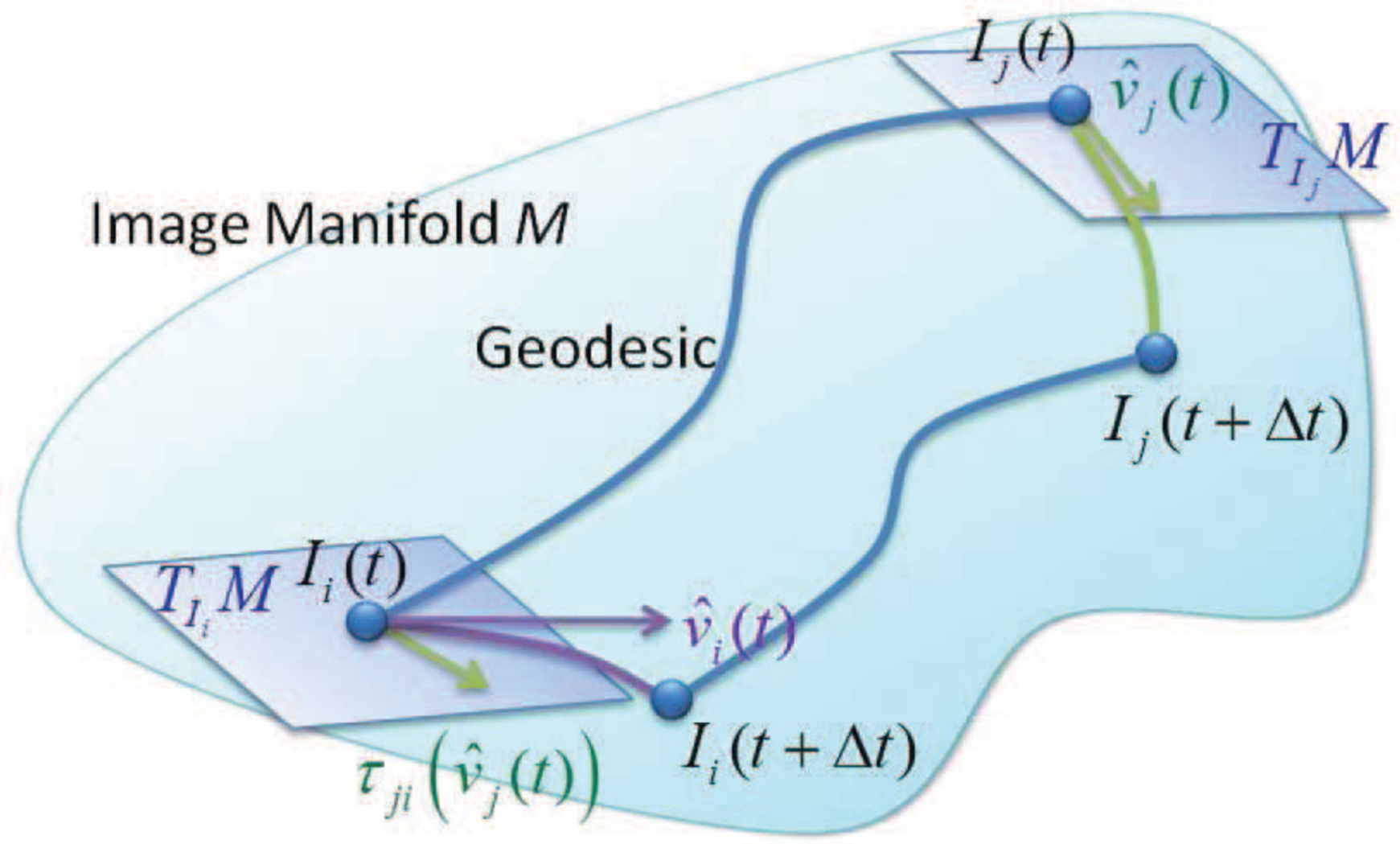

Figure 3.

Demonstration of the translation of a vector, and relationship between the current and next images. 


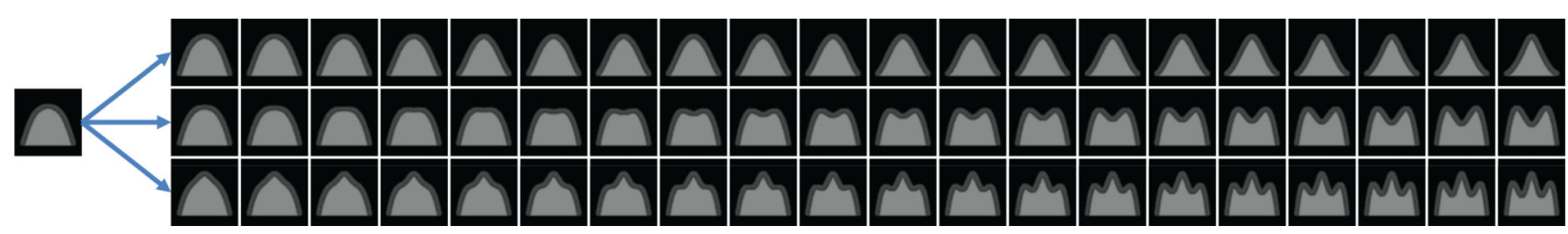

Figure 4.

A total of 61 images in the synthetic dataset. 


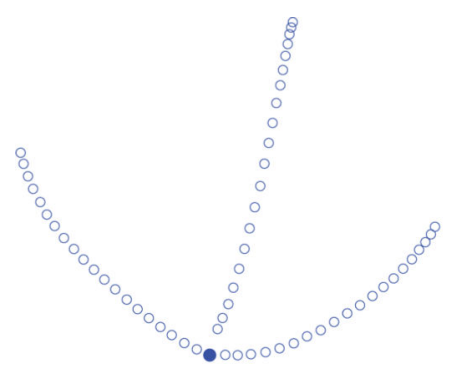

(a)

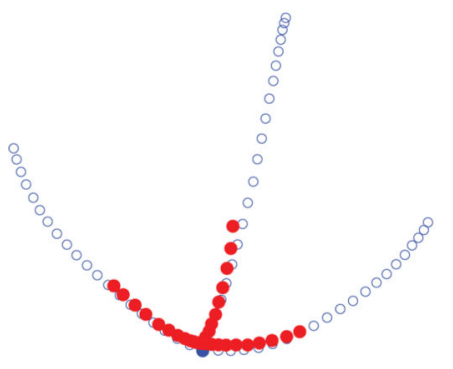

(d)

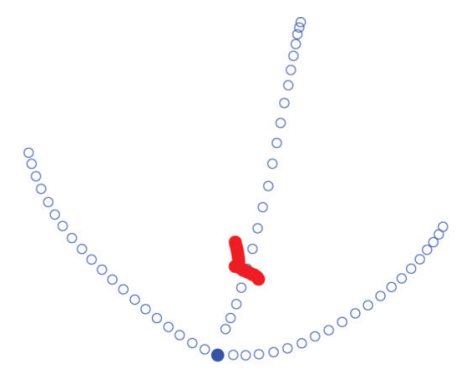

(b)

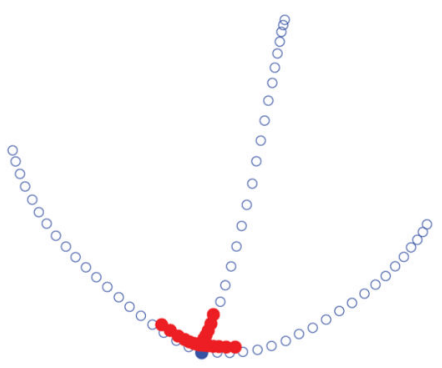

(e)

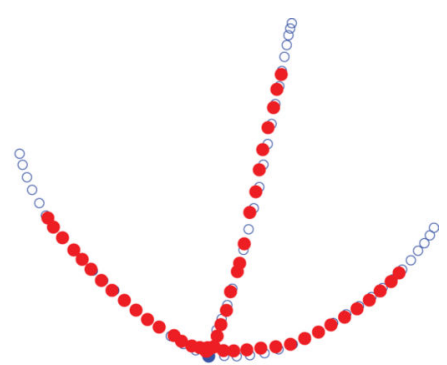

(c)

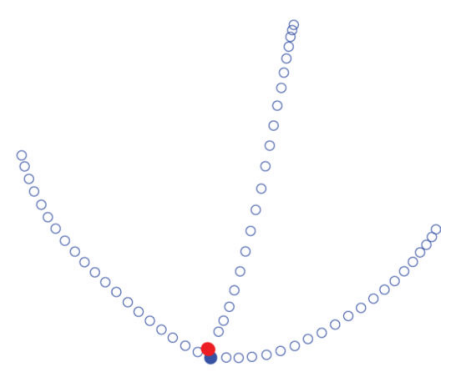

(f)

Figure 5 .

Registration process and registration results in $2 \mathrm{D}$ projected space. (a) The initial distribution, (b) registration result by conventional group-mean method, (c)-(e) the intermediate processes of registration by our method and (f) the final registration result by our method. 
\#1

\#2

\#3

\#4

\#5

\#6

\#7

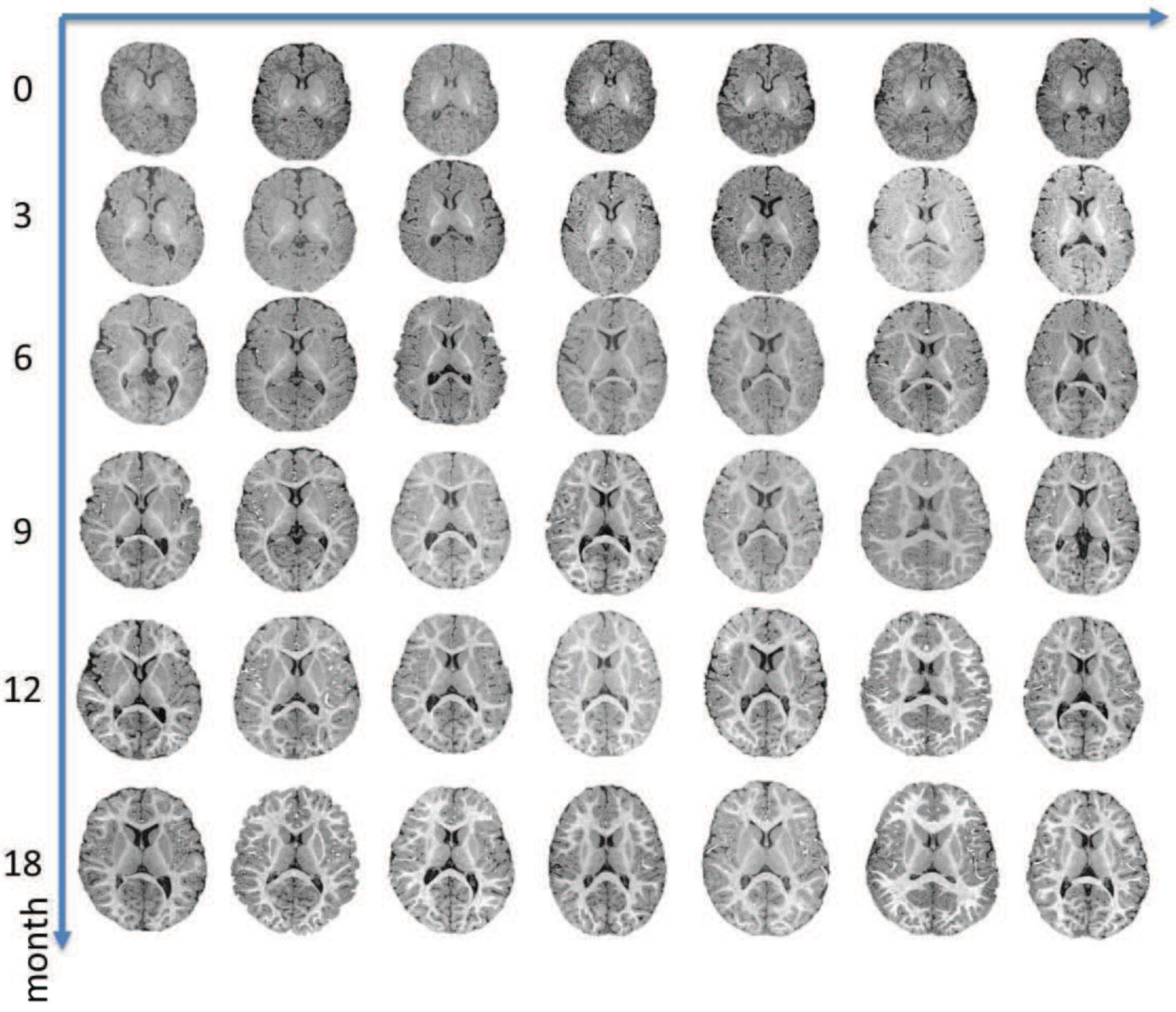

Figure 6.

A total of 42 images from longitudinal infant data set. 

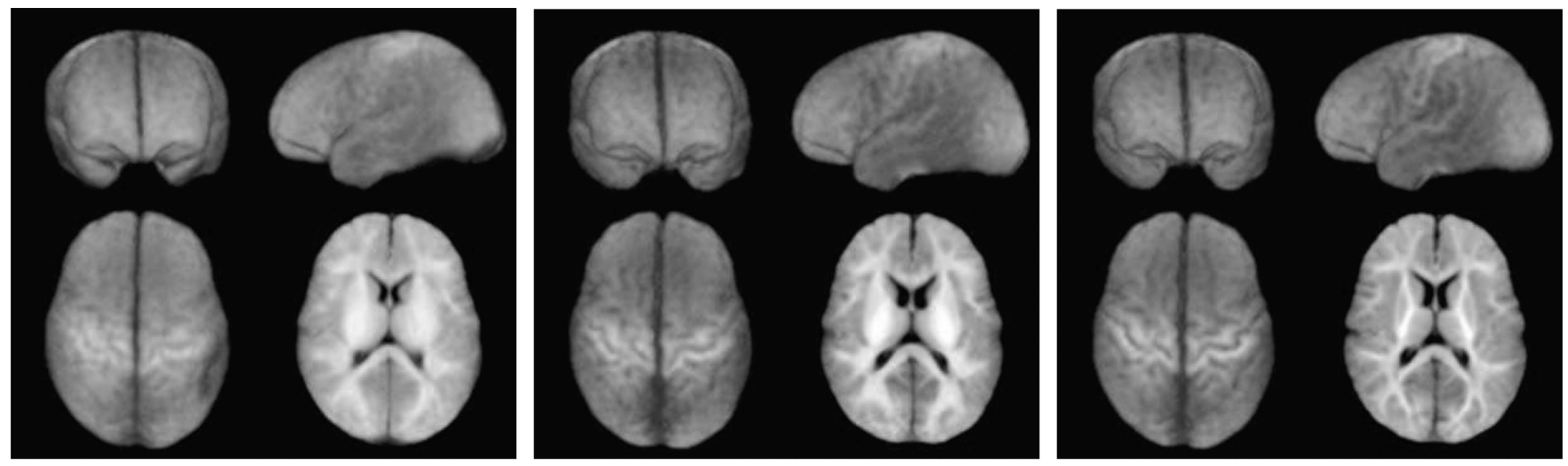
(a) Mean image by the
(b) Mean image by $\mathrm{AB}$
-(c) Mean image by our group-mean method SORB method

Figure 7.

Mean images by the three methods on longitudinal infant data set. 


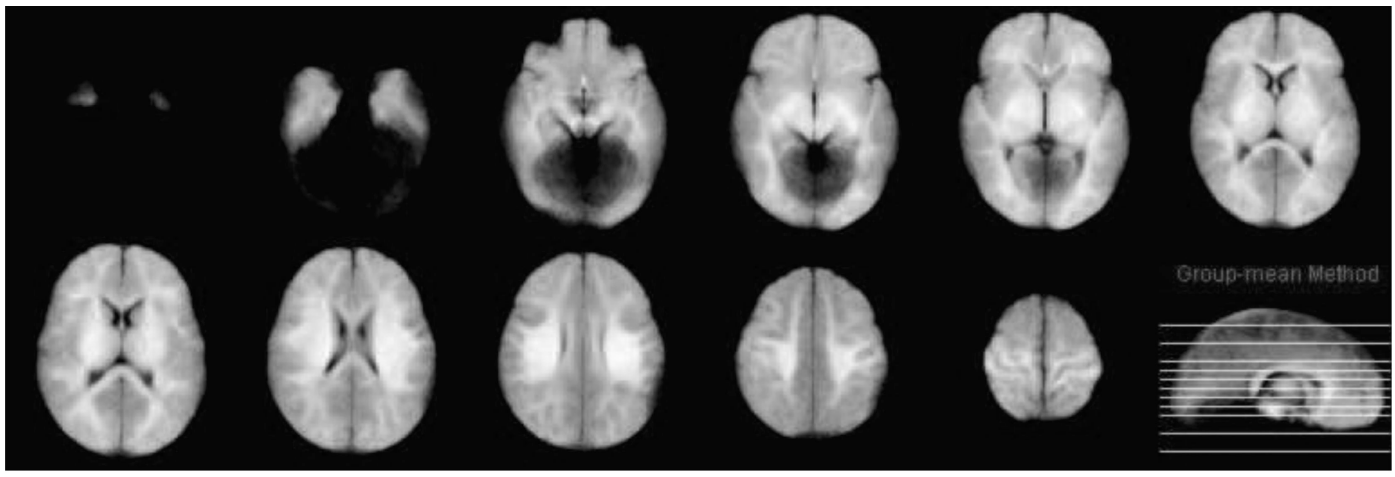

(a) Cross-sectional views of the mean image by the group-mean method

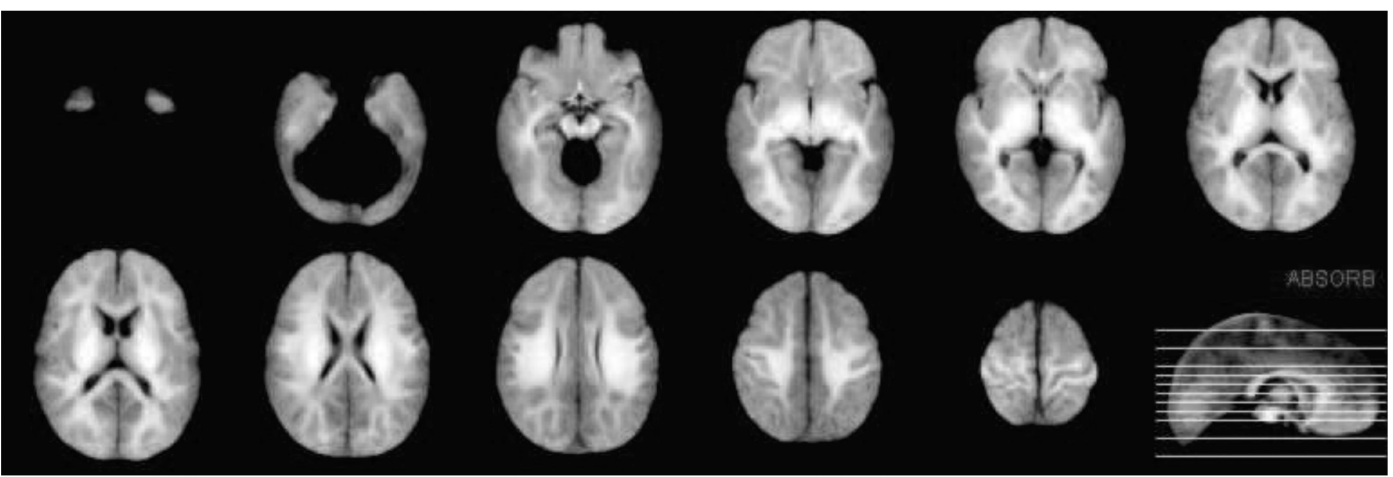

(b) Cross-sectional views of the mean image by ABSORB

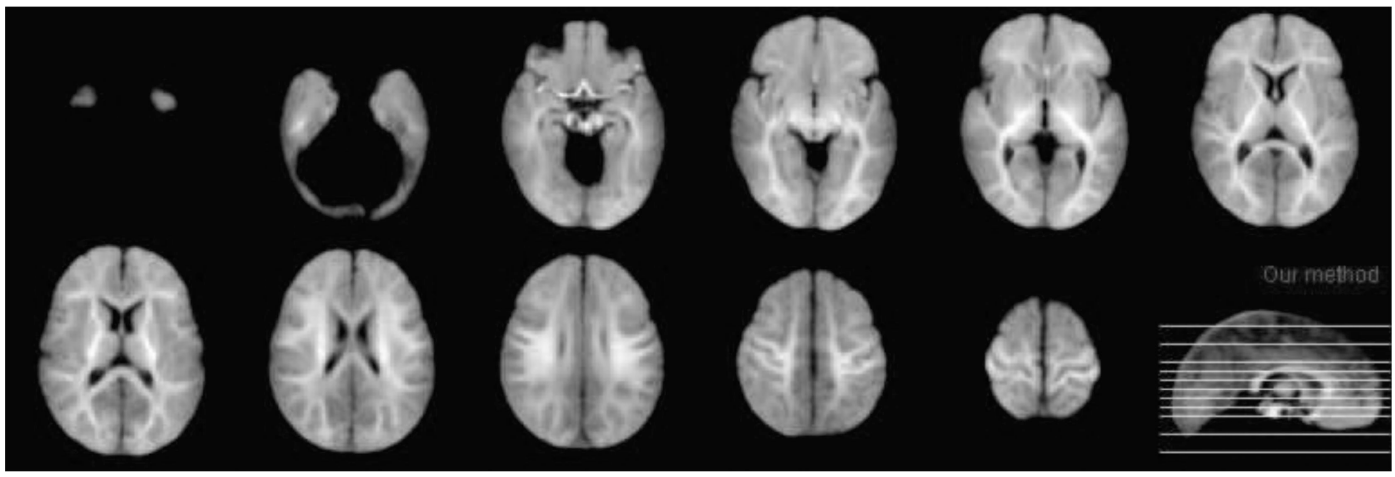

(c) Cross-sectional views of the mean image by our method

Figure 8.

Cross-sectional views of three mean images on longitudinal infant data set. 

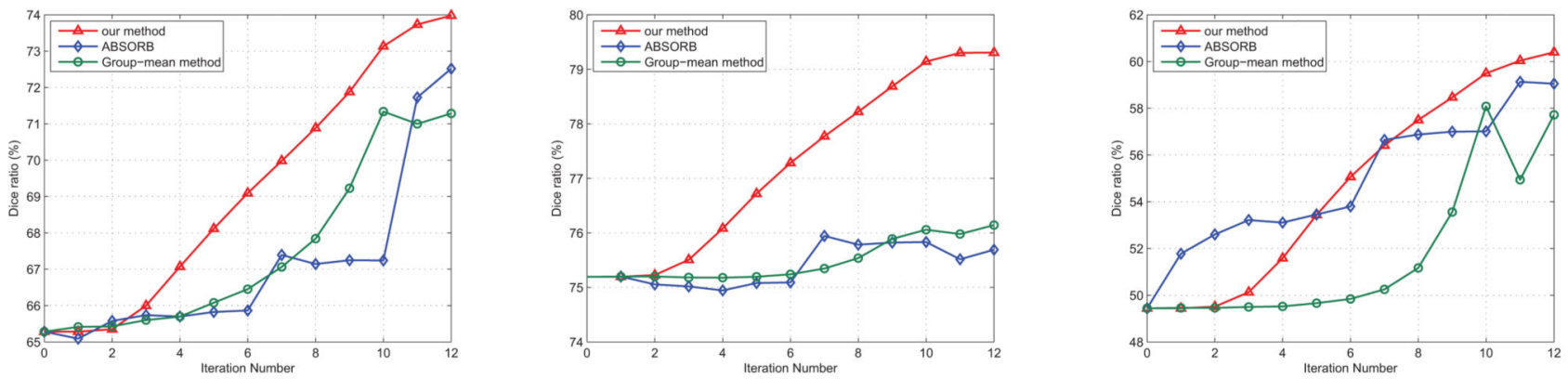

\section{(a) Evolution of Dice ratio (b) Evolution of Dice ratio (c) Evolution of Dice ratio on WM on GM on CSF}

Figure 9.

The evolution of the Dice ratios of three brain tissues during the groupwise registration by three methods on longitudinal infant data set. 


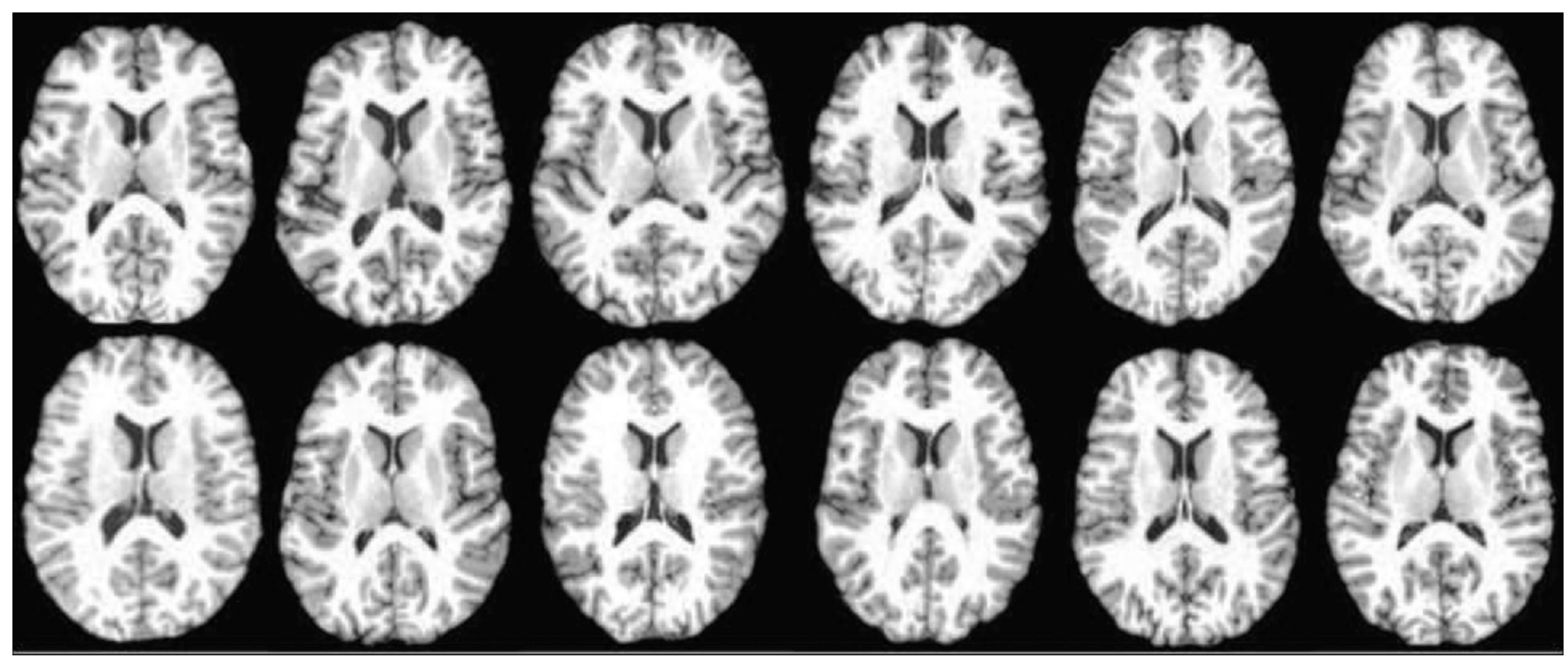

Figure 10.

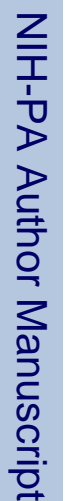

12 typical images from the IXI database. 


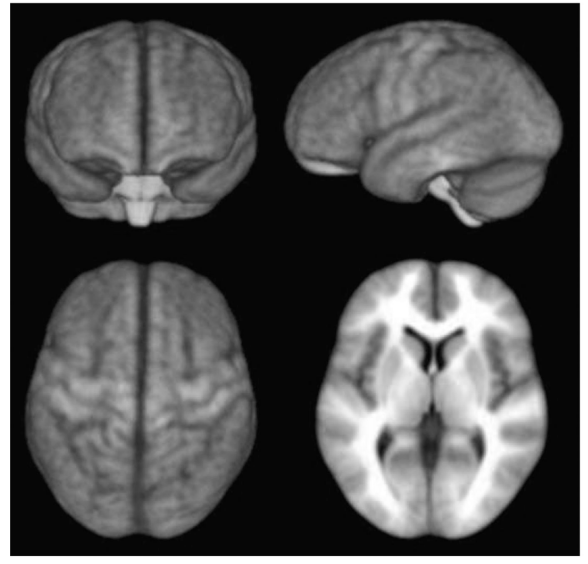

(a) Mean image by groupmean method

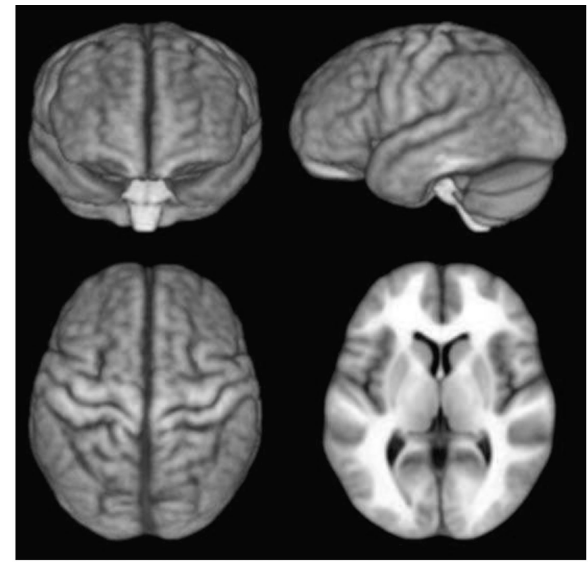

(b) Mean image by $\mathrm{AB}$ SORB

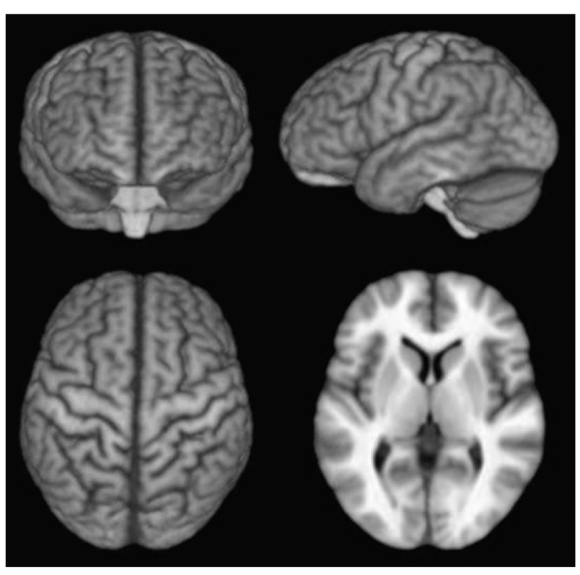

AB- (c) Mean image by our method

Figure 11.

Mean images by three methods on IXI data set. 


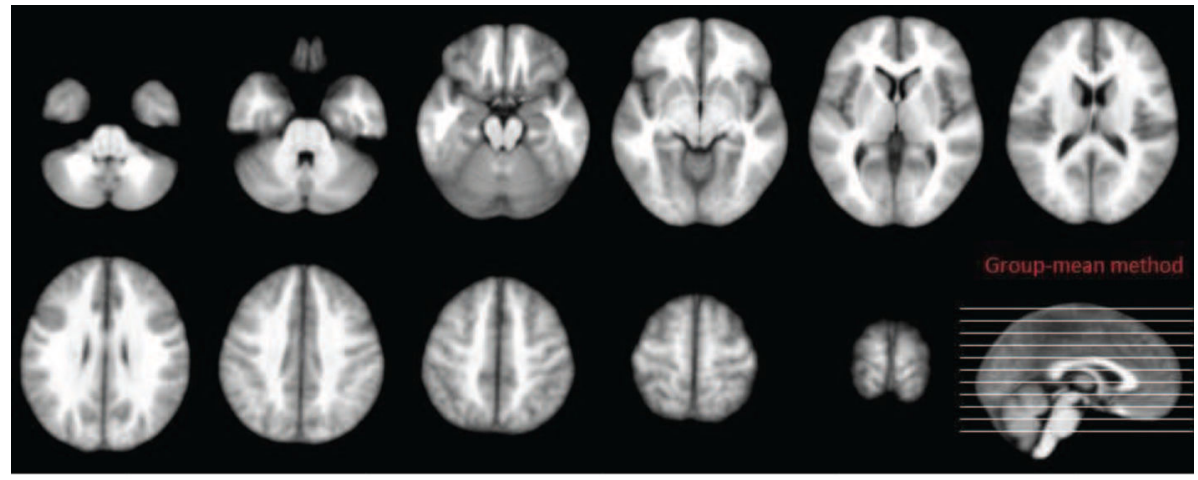

(a) Cross-sectional views of the mean image by group-mean method

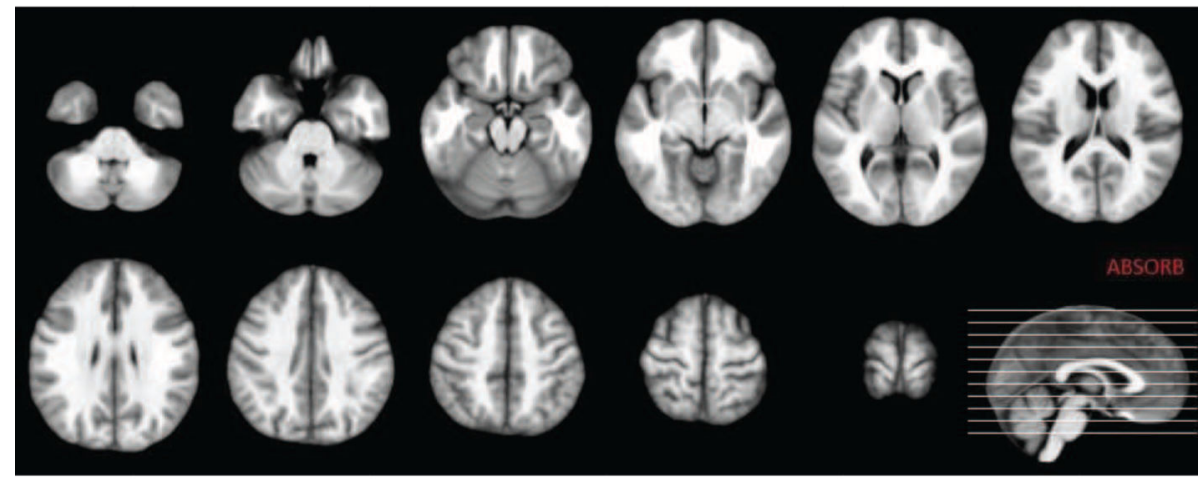

(b) Cross-sectional views of the mean image by ABSORB

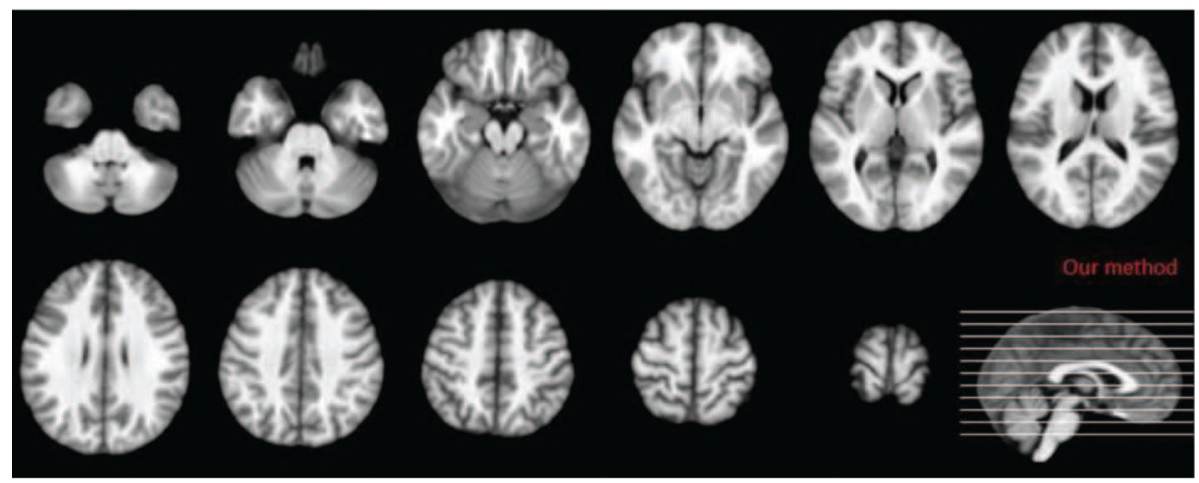

(c) Cross-sectional views of the mean image by our method

Figure 12.

Cross-sectional views of three mean images on IXI data set. 


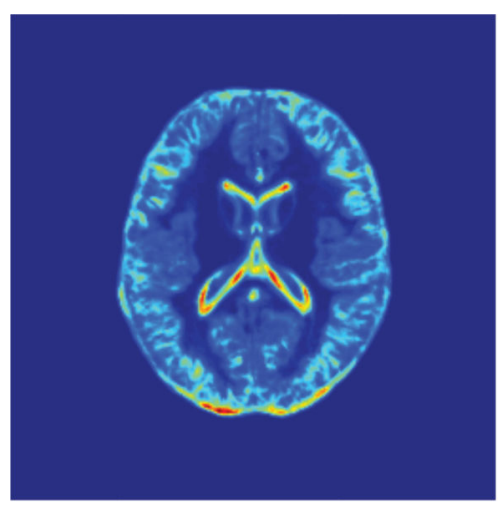

(a)

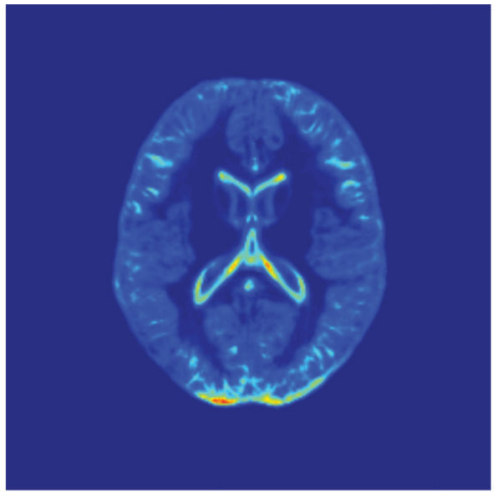

(b)

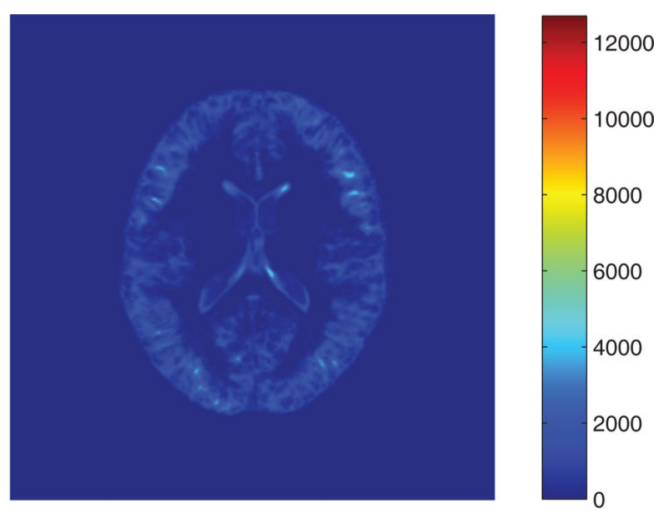

(c)

Figure 13.

The map of intensity difference between all warped image and the mean image on IXI data set. (a) Joshi's method, (b) ABSORB and (c) our method. 


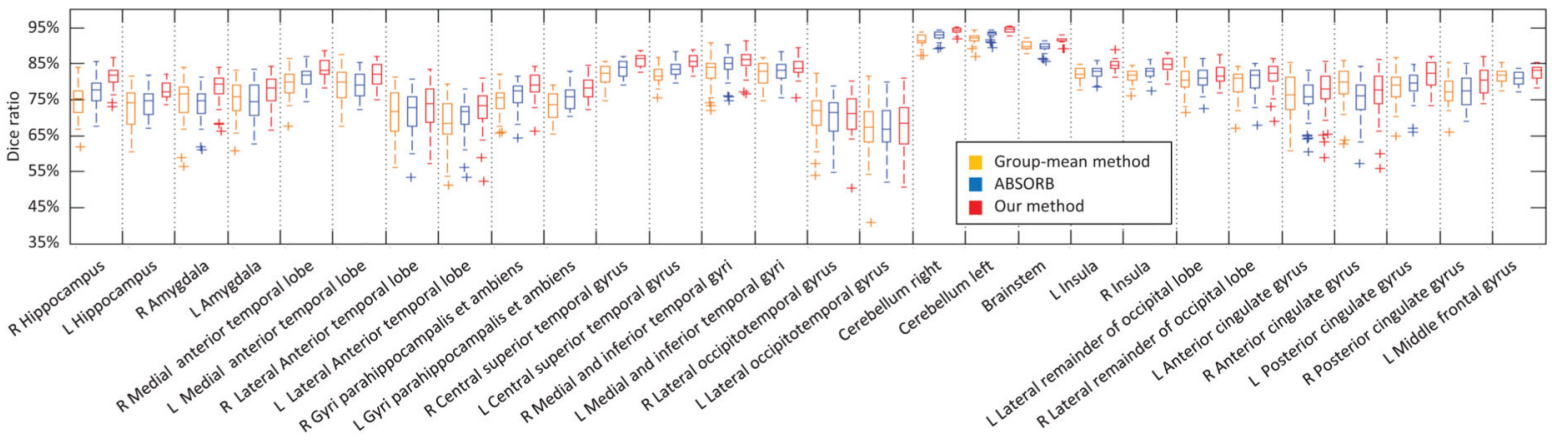

(a) Dice ratios of the 1st-28th ROIs by three methods

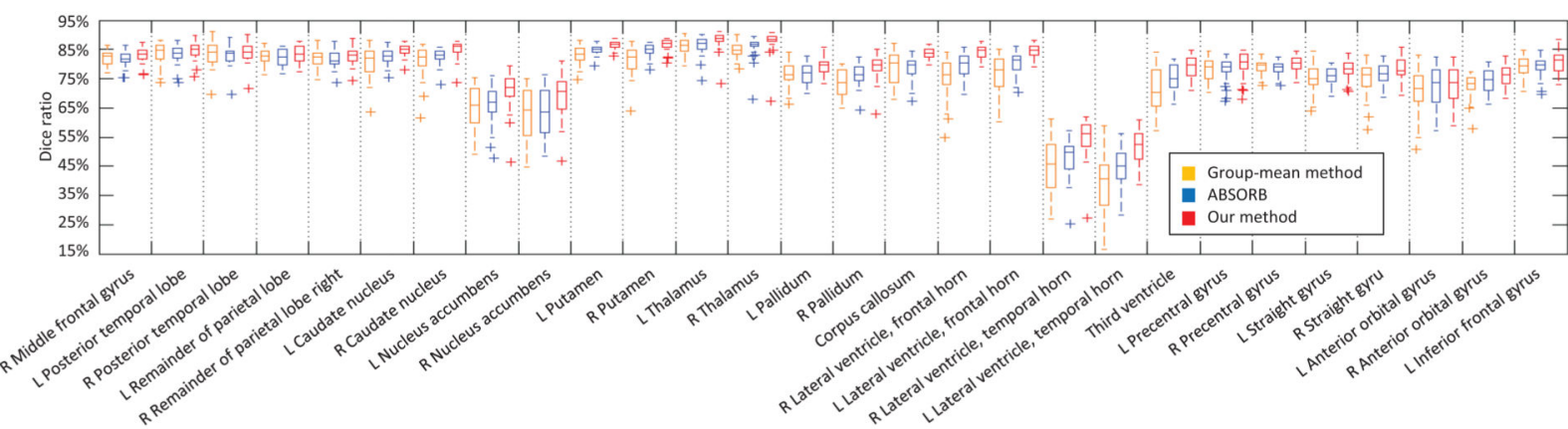

(b) Dice ratios of the 29 th- -56 th ROIs by three methods

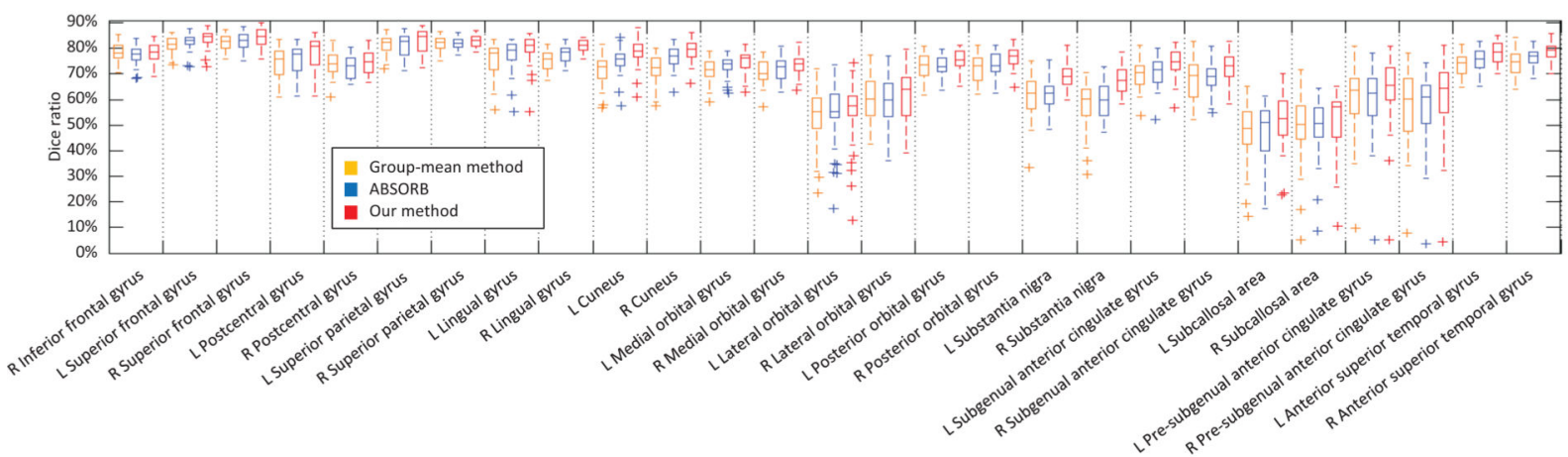

(c) Dice ratios of the 57th-83th ROIs by three methods

Figure 14.

Comparison of Dice ratios of 83 ROIs by three methods on IXI data set. 

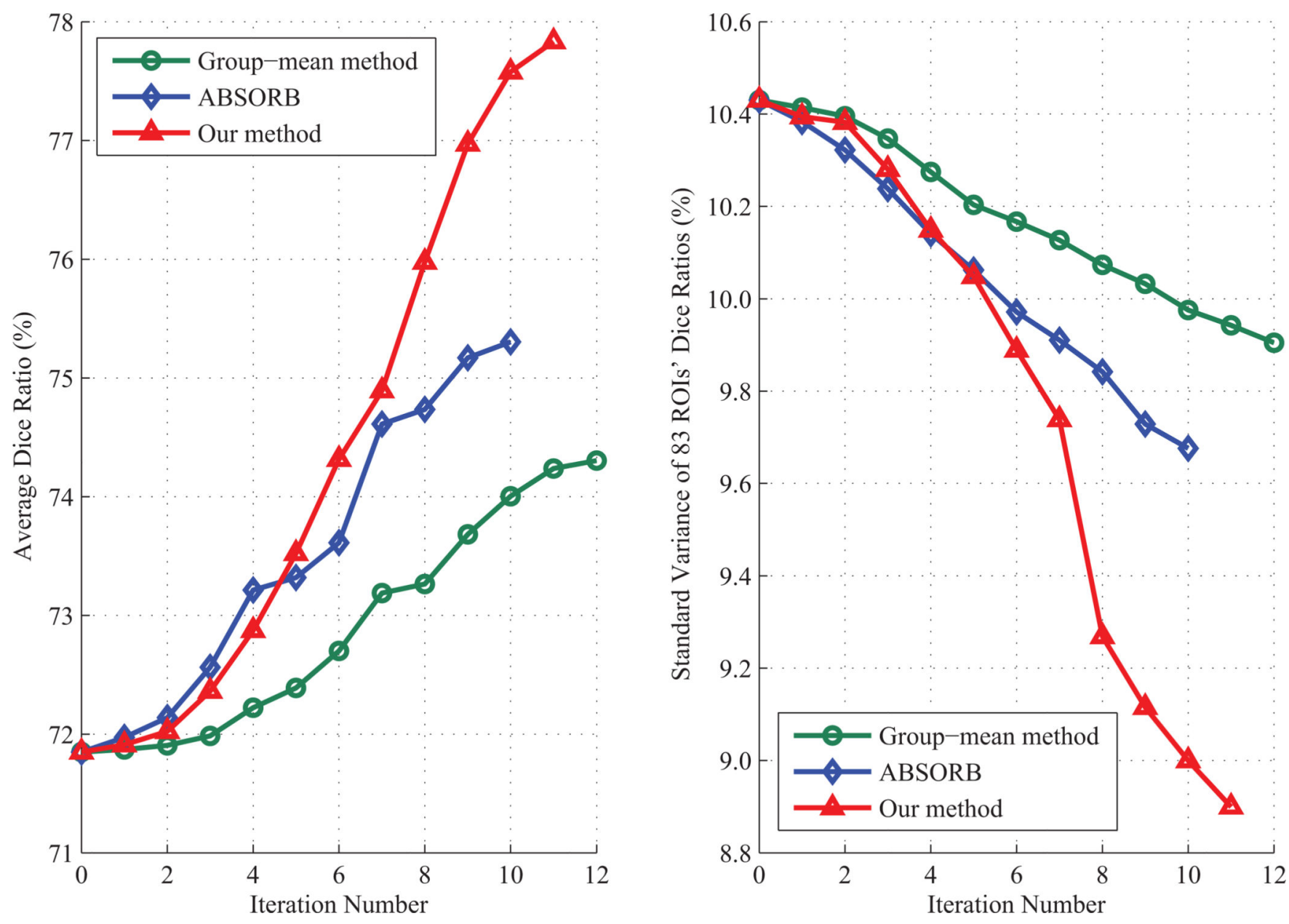

Figure 15.

The change of the average Dice ratio of 83 ROIs in IXI data, along with its respective standard deviation, with respect to the use of iterations in each of three different algorithms. 


\section{Table 1}

Dice ratios of three brain tissues by three methods

\begin{tabular}{lrrrr}
\hline & WM & GM & CSF & Overall \\
\hline Group-mean method & $71.28 \%$ & $76.14 \%$ & $57.71 \%$ & $68.38 \%$ \\
ABSORB & $72.51 \%$ & $75.69 \%$ & $59.05 \%$ & $69.09 \%$ \\
Our method & $\mathbf{7 3 . 9 8} \%$ & $\mathbf{7 9 . 3 1} \%$ & $\mathbf{6 0 . 3 9} \%$ & $\mathbf{7 1 . 2 3} \%$ \\
\hline
\end{tabular}

